\title{
Asset price dynamics when behavioural heterogeneity varies.
}

\author{
Domenico Colucci*and Vincenzo Valori*
}

November 2006

\begin{abstract}
We build a model in which asset prices are expectationally driven and agents forecast future prices hinging on a combination of fundamental value, trend and inertia. The model has a unique steady state and we investigate its stability. In particular the amount of behavioural heterogeneity in the model is given by the number of intermediaries actually operating in the market: we are concerned with the effects that changing such number produces on the steady state in terms of stability. Assuming that the set of relevant intermediaries is sampled randomly we discuss the probability of having stability as a function of the market's parameters and the number of such agents. Our simulations show that stability in the multi-agent setting does not require that conditions for stability in the representative agent case be met for every individual; so stability can arise even if some of the agents would not be compatible with it if they were the only ones operating in the market. The same goes for instability. Further, we find that stabilising (or destabilising) effects of heterogeneity are not uniform across the market's essential characteristics, as captured by a given structural parameter: in fact we can identify a parametric region in which heterogeneity is stabilising and another in which it is destabilising.

Journal of Economic Literature Classification Numbers: G12, D84.

Key Words: heterogeneous agents, expectations, stock market, behavioural finance, bounded rationality, middlemen.
\end{abstract}

\section{Introduction}

Behavioural heterogeneity is a rather well established phenomenon in financial markets from various standpoints: significant diversity in households' portfolio choices has been documented in several papers, among which Vissing-Jorgensen [16], as well as in the decisions to participate to stock markets. Heterogeneous expectations also seem to be the rule in financial markets, even though it is more difficult to actually observe expectations as such: Elliot and Ito [9] and Branch [4] for example present evidence of expectations heterogeneity in exchange rate markets and as regards inflation forecasts. The discussion contained in Frankel and Froot [11] clarifies the importance of such heterogeneity to explain the volume of trade exchange. Our previous paper [6] shows expectations heterogeneity in a Cobweb-type experimental context. Whether and to what extent this heterogeneity and its ultimate bounded rationality content matter remains controversial. The principal focus of this paper though is on a rather different aspect: we wish to understand the

*DIMAD - Dipartimento di Matematica per le Decisioni - Università degli Studi di Firenze - Via C. Lombroso, 6/17 - 50134 Firenze - Italy. E-mail: domenico.colucci@unifi.it, vincenzo.valori@dmd.unifi.it 
consequences of changes in the amount of heterogeneity in a simple asset pricing model, in particular with respect to the persistence of stability of equilibria. The reason for addressing this issue is that it can be an important feature arising in empirical contexts, such as when patterns of households direct participation in stock markets change (e.g. due to introduction of new technology, such as internet banking resources made available to the greater public) as compared to participation through the intermediation of a middleman; other situations in which large changes in the amount of agents' heterogeneity can show up include the merging of previously separated markets (e.g. when a free-trade area is created). Some related literature comprises Egenter et al. [8] who show that their model's ability to reproduce realistic time series properties vanishes if the number of traders goes to infinity, in the sense that the actions of different agents tend to compensate when their number increases therefore eliminating aggregate volatility. More recently Brock et al. [5] use the notion of large type limit to characterize persistent dynamical features of a heterogeneous agents model as the number of types is large.

Our model is in line with a thread of literature focusing on agents who have heterogeneous assessments of the future values of relevant economic variables which can be traced back to Beja and Goldman [1]. Specifically we build on Day and Huang [7] introducing a different baseline type of expectations mechanism which is a combination of trend-chasing and fundamentalist behaviour plus an inertia component taking an adaptive form. We also allow heterogeneity in the beliefs, which pushes up the dimensionality of the resulting dynamics.

The paper is organised as follows: in section 2 we introduce the baseline model, i.e. the model with a single intermediary operating on the market and derive conditions for local stability of its unique steady state. In section 3 we introduce behavioural heterogeneity in the form of a multiplicity of middlemen and compute the probability of having a stable steady state in the baseline case when the intermediary is selected at random. Section 4 describes our simulations, whose results are discussed in Section 5 which includes some concluding remarks.

\section{The baseline model}

We carry out our analysis on a model of stock market behaviour heavily inspired by the seminal Day and Huang 1990's paper (see [7]). We consider a representative investor whose excess demand linearly depends on the difference between expectations about future prices and actual present price:

$$
Z_{t}\left(p_{t+1}^{e}, p_{t}\right)=\sigma\left(p_{t+1}^{e}-p_{t}\right)
$$

where $p_{t}$ is the stock price at time $t, p_{t+1}^{e}$ is the investor's forecast of prices at time $t+1$ and $\sigma$ is a positive coefficient. A market maker sets the price in response to aggregate excess demand and saturates the market when it is out of the equilibrium by reducing or increasing his inventory of stocks. Just to keep the inventory in balance, prices are set in a tatonnement-like process, increasing when the inventory reduces and vice-versa. We consider the linear price adjustment function

$$
p_{t+1}=F\left(p_{t+1}^{e}, p_{t}\right)=p_{t}+\lambda Z_{t}\left(p_{t+1}^{e}, p_{t}\right)
$$

where the parameter $\lambda>0$ sets the adjustment sensitivity.

\subsection{Expectations}

Although in the real world price expectations depend on a multiplicity of factors, in what follows we suppose that only three determinants are at work: inertia, fundamental value and trend. While 
fundamentalists/chartists types are standard in asset pricing models with heterogeneous agents, we also suppose that a certain amount of inertia can play a role. This is suggested by several papers: on the theoretical side, Morris and Shin [13] show that forward-looking expectations imply inertia (behaving like adaptive expectations) when information on fundamentals is heterogeneous across individuals; Hommes et al. [12] on the other hand implement an experimental asset pricing model and observe that a significant fraction of agents employ some forms of adaptive beliefs to predict future prices; Benassy-Quére et al. [2] use adaptive, extrapolative (i.e. chartist) and regressive models in their econometric study of expectations heterogeneity in a panel of data from the (exchange rate) forecasts of Consensus Economics.

The three building blocks of our updating rule are specified as follows. For the inertia component we take a convex combination of last observed price and expectations, $\alpha p_{t}^{e}+(1-\alpha) p_{t}$. We also assume that investors have some perception of the fundamental value, $v$, of the asset under consideration and that if they observe a price greater then $v$ then they will expect a capital loss, at least in the medium/long run. Further, the perceived probability that $p$ will decline in the next future increases with the value of $p$. This part of the model is very much like Day and Huang's. But while they define a weighting function measuring (in their words) the chance of lost opportunity and incorporate it in the, otherwise linear, excess demand function, we directly define expectations dependence by the fundamental value in a non-linear way which preserves the qualitative properties of the original model. Such dependence is modeled by the cubic function $\beta\left(\left(p_{t}-v\right)+\left(p_{t}-v\right)^{3}\right)$. Finally, we suppose that agents incorporate last observed price movement in their forecast of future price. If the price increased/decreased in the last period, investors will think that such trend will be preserved, at least partially, in the near future. This trend following term is taken to be linear and equal to $\gamma\left(p_{t}-p_{t-1}\right)$. With this specification we have that the fundamentalist part is dominant when prices are far from the fundamental value. Conversely, when prices are near the equilibrium, the trend following and the fundamentalist components could reinforce or balance each other; in the latter case the dominance is determined by the greater between the two coefficients $\beta$ and $\gamma$. Putting everything together expectations become

$$
p_{t+1}^{e}=\alpha p_{t}^{e}+(1-\alpha) p_{t}-\beta\left(\left(p_{t}-v\right)+\left(p_{t}-v\right)^{3}\right)+\gamma\left(p_{t}-p_{t-1}\right)
$$

with $\beta, \gamma \geq 0$ and $\alpha \in[0,1]$. The above general form clearly encompasses simple rules when one or more parameters equal zero.

By substituting (3) in (1) and then in turn in (2) we get the equation for prices. The model, setting $\delta=\lambda \sigma$, can then be written as a first order three-dimensional system:

$$
\left\{\begin{array}{l}
p_{t+1}=p_{t}+\delta\left(\alpha p_{t}^{e}+(1-\alpha) p_{t}-\beta\left(\left(p_{t}-v\right)+\left(p_{t}-v\right)^{3}\right)+\gamma\left(p_{t}-x_{t}\right)-p_{t}\right) \\
p_{t+1}^{e}=\alpha p_{t}^{e}+(1-\alpha) p_{t}-\beta\left(\left(p_{t}-v\right)+\left(p_{t}-v\right)^{3}\right)+\gamma\left(p_{t}-x_{t}\right) \\
x_{t+1}=p_{t}
\end{array}\right.
$$

whose unique steady state is $\left(p^{*}, p^{e *}, x^{*}\right)=(v, v, v)$. In the next Proposition we summarize the local stability properties of the unique steady state of the system (4). 
Proposition 1 Given the system (4) its unique steady state E is locally stable iff

$$
\begin{aligned}
& \frac{\delta\left(\gamma-\frac{\beta}{2}\right)+1}{\delta-1}<\alpha<\frac{\gamma \delta-1}{\delta-1} \text { when } \delta<1 \\
& \frac{\beta}{2}-1<\gamma<1 \text { when } \delta=1 \\
& \frac{\gamma \delta-1}{\delta-1}<\alpha<\frac{\delta\left(\gamma-\frac{\beta}{2}\right)+1}{\delta-1} \text { when } \delta>1 .
\end{aligned}
$$

Period-doubling bifurcations occur along the lines $\alpha=\frac{\delta\left(\gamma-\frac{\beta}{2}\right)+1}{\delta-1}$ and $\gamma=\frac{\beta}{2}-1$, while NeimarkSacker bifurcations occur along the lines $\alpha=\frac{\delta \gamma-1}{\delta-1}$ and $\gamma=1$.

Proof. The Jacobian, evaluated at the steady state is

$$
J(v, v, v)=\left(\begin{array}{ccc}
1-\delta \alpha-\delta \beta+\delta \gamma & \delta \alpha & -\delta \gamma \\
1-\alpha-\beta+\gamma & \alpha & -\gamma \\
1 & 0 & 0
\end{array}\right)
$$

Its characteristic polynomial is

$$
X^{3}+(\alpha \delta+\beta \delta-\gamma \delta-\alpha-1) X^{2}+(\alpha-\alpha \delta+\gamma \delta) X
$$

So, one eigenvalue is $X=0$. To discuss stability properties of the system one can use the trace/determinant conditions

$$
\left\{\begin{array} { l } 
{ D < 1 } \\
{ T < 1 + D } \\
{ T > - 1 - D }
\end{array} \Rightarrow \left\{\begin{array}{l}
\alpha-\alpha \delta+\gamma \delta<1 \\
-\alpha \delta-\beta \delta+\gamma \delta+\alpha+1<1+\alpha-\alpha \delta+\gamma \delta \\
-\alpha \delta-\beta \delta+\gamma \delta+\alpha+1>-1-(\alpha-\alpha \delta+\gamma \delta)
\end{array}\right.\right.
$$

The second equation reduces to

$$
\beta \delta>0
$$

and is always satisfied in the considered parameter space, while the first and third equations, which determine Neimark-Sacker and Period-doubling bifurcations respectively, give the claimed result.

Figures 1 to 3 show some examples of the bifurcation diagram with respect to the parameters $(\gamma, \alpha)$ when $\beta$ or $\delta$ vary. The grey area is the stable parameter region; the thick line refers to the Neimark bifurcation while the thin one (which appears only in Figure 2 and 3 ) is that of the Perioddoubling bifurcation. Dashed lines show movements of the bifurcation lines when $\beta$ or $\delta$ change. For $\delta<1$, if $\beta<2$, which means there can be up to $200 \%$ response to the observed deviation, only a Neimark bifurcation is possible (see Figure 1). When $\delta>1$ both bifurcations are possible. The stability area shrinks when either $\delta$ or $\beta$ increase. On the contrary, there is not a clear effect on the size of the stability area when $\alpha$ increases, while $\gamma$ has a non-monotonic impact.

[Figures 1, 2 and 3 about here] 


\section{$3 \quad$ Intermediaries and market heterogeneity}

The role of middlemen has been extensively studied under different points of view. Their role in facilitating trade when some market imperfections are at work has attracted deep attention (see for example Johri and Leach [14]); in particular Biglaiser [3] discusses the topic in the context of adverse selection and moral hazard. Other examples include Fingleton [10] who addresses the effect of disintermediation policies on middlemen's market power and Rust and Hall [15] who study a market model in which a market-maker and middlemen coexist as competing intermediaries.

We aim at inquiring about dynamic implications due to the presence of intermediaries in the market. The emphasis is not on the abilities of this particular class of - presumably more informed agents but simply on the consequences on the economic dynamics of having one or more middlemen who mediate investors' access to the market. The way we introduce behavioural heterogeneity in the above model entails considering the homogeneous version as the (admittedly rather extreme) case in which a single intermediary acts on behalf of a given population of traders. We assume that heterogeneity is introduced when more than one middleman operates on the market, whereas the population of traders and the potential amount of wealth that is invested in the market stay the same. We simply assume that the agents acting as middlemen are randomly selected over the population. Indeed, drawing a larger sample of agents implies a higher expected behavioural variability: therefore it is not unreasonable to consider the number of relevant intermediaries as a proxy for the degree of expectation heterogeneity. This is also a way to disentangle the effects of behavioural diversity from the problems of aggregation to which it is usually intertwined. Clearly, the model can also be considered as one in which the economy's fundamental characteristics are constant with the only exception of the number of different agents (and therefore the degree of behavioural diversity) operating, ceteris paribus.

\subsection{Effect of heterogeneity on stability}

There are obviously several ways of defining the concept of stabilising (or destabilising) heterogeneity: our perspective in this paper is to consider each agent as a potential intermediary characterised by a given vector of expectational parameters (namely $\alpha, \beta$ and $\gamma$ ). Then the probability of drawing an $n$-tuple of intermediaries from the population such that the system's equilibrium in which these agents act as middlemen is locally stable given the parameters of the economy (basically $\delta$ ), can, in principle, be computed as a function of $n$. When this probability increases with $n$ we shall say that heterogeneity is stabilising and vice versa. It turns out, as we show below, that in this model we do not get a form of stabilising or destabilising heterogeneity irrespective of the economy's characteristics. Instead, for given values of $\delta$ heterogeneity can be stabilising while for other values of $\delta$ the situation is reversed. An intuition for this result rests on the meaning of the parameter $\delta$, which is an aggregate measure of sensitivity of the price to excess demand and to expected price change.

First of all we can compute the probability of having a locally stable steady state for the baseline model when its parameters $(\alpha, \beta, \gamma)$ are assumed to be drawn from a uniform distribution. To this end we must bound the parameter space. In the model settings we have supposed that $\alpha \in[0,1]$ and $\beta, \gamma \geq 0$, so an upper bound for $\beta$ and $\gamma$ remains to be set. In the following discussion we shall require $\beta, \gamma \leq 2$ assuming that agents reaction will never be greater than $200 \%$ of the price difference they refer to. It turns out that this particular value for the upper bound is not crucial in terms of the implied qualitative properties of how the probability of local stability is related to $\delta$. We develop on this robustness issue in Section 5 and in the Appendix. 
Proposition 2 Consider the baseline model described in equation (4) and suppose that the triple $(\alpha, \beta, \gamma)$ be the realization of a random variable with uniform distribution in $[0,1] \times[0,2] \times[0,2]$. Then the probability for the steady state of being locally stable and hyperbolic is

$$
P(\delta)=\left\{\begin{array}{clc}
\frac{5 \delta-4}{4 \delta-4} & \text { if } & 0<\delta \leq \frac{1}{2} \\
\frac{1+\delta}{4 \delta} & \text { if } & \frac{1}{2}<\delta \leq 1 \\
\frac{-4 \delta^{2}+17 \delta-7}{12 \delta^{2}} & \text { if } & 1<\delta \leq 2 \\
\frac{12 \delta-13}{12 \delta^{2}(\delta-1)} & \text { if } & \delta>2
\end{array}\right.
$$

Proof. Conditions (5) can be equivalently written as

$$
\begin{aligned}
& \beta<\frac{2+2 \delta \gamma-2 \alpha(\delta-1)}{\delta} \triangleq P D_{\delta}(\gamma, \alpha) \text { and } \gamma<\frac{1+\alpha(\delta-1)}{\delta} \triangleq N S_{\delta}(\alpha) \text { if } \delta \neq 1 \\
& \beta<2 \gamma+2 \text { and } \gamma<1 \text { if } \delta=1
\end{aligned}
$$

Then, the probability of having a stable system for a given $\delta>0$ is

$$
P(\delta)=\frac{1}{4} \int_{0}^{1} \int_{0}^{\max \left\{0, \min \left\{N S_{\delta}(\alpha), 2\right\}\right\}} \max \left\{0, \min \left\{P D_{\delta}(\gamma, \alpha), 2\right\}\right\} d \gamma d \alpha
$$

Notice that $N S_{\delta}(\alpha)>0$ for any $\delta>0$, so $\max \left\{0, \min \left\{N S_{\delta}(\alpha), 2\right\}\right\}=\min \left\{N S_{\delta}(\alpha), 2\right\}$ in all cases. When $\delta<1$ only the constraint $\gamma<N S_{\delta}(\alpha)$ is active and the probability of having a stable steady state is

$$
\begin{aligned}
P(\delta) & =\frac{1}{4} \int_{0}^{1} \int_{0}^{\min \left\{N S_{\delta}(\alpha), 2\right\}} 2 d \gamma d \alpha= \\
& =\left\{\begin{array}{lll}
\frac{1}{4} \int_{0}^{\frac{2 \delta-1}{\delta-1}} \int_{0}^{2} 2 d \gamma d \alpha+\frac{1}{4} \int_{\frac{2 \delta-1}{\delta-1}}^{1} \int_{0}^{N S_{\delta}(\alpha)} 2 d \gamma d \alpha & \text { if } & 0<\delta<\frac{1}{2} \\
\frac{1}{4} \int_{0}^{1} \int_{0}^{N S_{\delta}(\alpha)} 2 d \gamma d \alpha & \text { if } & \frac{1}{2} \leq \delta<1
\end{array}\right. \\
& =\left\{\begin{array}{lll}
\frac{5-4}{4-4} & \text { if } & 0<\delta<\frac{1}{2} \\
\frac{1+\delta}{4 \delta} & \text { if } & \frac{1}{2} \leq \delta<1
\end{array}\right.
\end{aligned}
$$

Note also that $P(1)=\frac{1}{2}$. When $\delta>1$ both constraints are active and we have

$$
\begin{aligned}
P(\delta) & =\frac{1}{4} \int_{0}^{1} \int_{0}^{\min \left\{N S_{\delta}(\alpha), 2\right\}} \max \left\{0, \min \left\{P D_{\delta}(\gamma, \alpha), 2\right\}\right\} d \gamma d \alpha= \\
& =\left\{\begin{array}{clc}
\frac{-4 \delta^{2}+17 \delta-7}{12 \delta^{2}} & \text { if } & 1<\delta \leq 2 \\
\frac{12 \delta-13}{12 \delta^{2}(\delta-1)} & \text { if } & \delta>2
\end{array}\right.
\end{aligned}
$$

which $^{1}$ completes our proof.

\footnotetext{
${ }^{1}$ Indeed, observing that from $P D(\gamma, \alpha, \delta)=2$ and $P D(\gamma, \alpha, \delta)=0$ we obtain $\gamma=\frac{-2 \alpha+2 \delta+2 \alpha \delta-2}{2 \delta}$ and $\gamma=$ $\frac{-\alpha+\alpha \delta-1}{\delta}$ respectively, $P(\delta)$ writes as

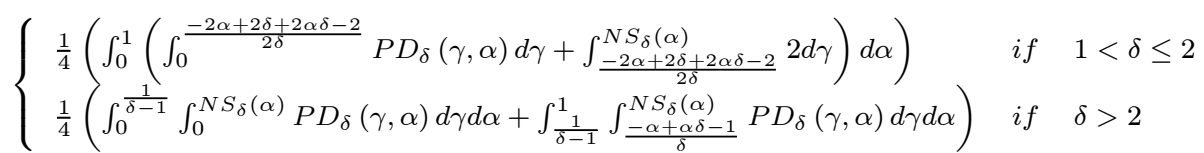

which can be simplified as above. 
Observe that the analytical expression above is continuous and decreasing in $\delta$ : more price sensitivivity to excess demand and expected price change implies a less likely stable outcome as a result of a random selection of a middleman.

\subsection{The model with behavioural heterogeneity}

We build on the baseline model considering a set of $n$ intermediaries whose excess demand is proportional to a given endowment - say the amount of available wealth, which we suppose summing up to one over the whole population and being constant across agents and across time - and linearly depends on the difference between private expectations and last observed price

$$
Z_{t}^{(i)}\left(p_{t+1}^{(i)}, p_{t}\right)=\frac{\sigma}{n}\left(p_{t+1}^{(i)}-p_{t}\right) \quad i=1, \ldots, n
$$

where $p_{t+1}^{(i)}$ is the i-th investor's forecast of prices at time $t+1$. The aggregate excess demand is

$$
Z_{t}\left(p_{t+1}^{(1)}, \ldots, p_{t+1}^{(n)}, p_{t}\right)=\sum_{i=1}^{n} Z_{t}^{(i)}\left(p_{t+1}^{(i)}, p_{t}\right)
$$

and, similar to the baseline model, the linear price adjustment function is

$$
p_{t+1}=F\left(p_{t+1}^{(1)}, \ldots, p_{t+1}^{(n)}, p_{t}\right)=p_{t}+\lambda Z_{t}\left(p_{t+1}^{(1)}, \ldots, p_{t+1}^{(n)}, p_{t}\right)
$$

Heterogeneity resides in the expectation function which we assume to admit different parameter specifications across our population

$$
p_{t+1}^{(i)}=\alpha^{(i)} p_{t}^{(i)}+\left(1-\alpha^{(i)}\right) p_{t}-\beta^{(i)}\left(\left(p_{t}-v\right)^{3}+\left(p_{t}-v\right)\right)+\gamma^{(i)}\left(p_{t}-p_{t-1}\right)
$$

Substituting (8) in (6) and (6) in (7) and setting $\delta=\lambda \sigma$ we have

$$
\left\{\begin{array}{l}
p_{t+1}=p_{t}+\delta\left(\sum_{i=1}^{n} \frac{\alpha^{(i)} p_{t}^{(i)}+\left(1-\alpha^{(i)}\right) p_{t}-\beta^{(i)}\left(\left(p_{t}-v\right)^{3}+\left(p_{t}-v\right)\right)+\gamma^{(i)}\left(p_{t}-x_{t}\right)}{n}-p_{t}\right) \\
p_{t+1}^{(i)}=\alpha^{(i)} p_{t}^{(i)}+\left(1-\alpha^{(i)}\right) p_{t}-\beta^{(i)}\left(\left(p_{t}-v\right)^{3}+\left(p_{t}-v\right)\right)+\gamma^{(i)}\left(p_{t}-x_{t}\right) \\
x_{t+1}=p_{t}
\end{array}\right.
$$

Our perspective here focuses on the effects of changes in $n$ on the dynamics and in particular on the stability properties of the steady state. The way the model is framed in system (9) ensures that changing $n$ does not produce any change in the aggregate dimension of the market, because the total wealth invested (which belongs to the population on whose behalf the mediators operate) is constant over time.

\section{Simulations.}

We ran two sets of simulations to assess the effects of changes in the amount of heterogeneity as measured by the number of agents actually operating as intermediaries.

First, we carried a Monte Carlo simulation to evaluate the probability of having a locally stable steady state in our model as the number of these agents, $n$, increases. In particular for various 
$n$ and for a given grid of values of $\delta$ we have simulated economies by repeatedly sampling triples of parameters $\left(\alpha^{(i)}, \beta^{(i)}, \gamma^{(i)}\right)$ for each agent. Given $n$, for each $\delta$ at each repetition we kept track of whether or not the spectral radius of the resulting Jacobian matrix (i.e. the largest of the eigenvalues' moduli) evaluated at the steady state was less than one. Then probabilities were computed as relative frequencies of cases in which the spectral radius was less than one over the number of throws. In particular the specific settings in our simulations were as in Table 1.

[Table 1 about here]

The next set of simulations serves the purpose of clarifying the possible effects of merging two different economies: in particular, beside checking the intuitive results concerning the merging of two (un)stable economies (which unsurprisingly result in a larger (un)stable economy), we wanted to investigate the possibilities of stabilising/destabilising mergers. We repeatedly generated couples of economies manned with respectively $n_{1}$ and $n_{2}$ agents whose parameters were randomly drawn using the same specifications as above (with a common value for $\delta$ ) and $n_{1}, n_{2}$ both random (independent) integers between 1 and 50 .

\section{Discussion and conclusions}

Our results are summarised in Figures 4, 5 and Table 2.

\section{[Figure 4 about here]}

First observe that the way the collection of curves in Figure 4 are organised ${ }^{2}$ implies that there is a region (for $\delta$ below a certain threshold) in which heterogeneity is stabilising: increasing $n$ the probability of drawing a sample that entails stability of the steady state increases. There is also a region (for $\delta$ above a certain threshold) in which heterogeneity destabilises: increasing $n$ reduces the probability of samples that imply stability of the steady state. There is a sort of polarising effect due to heterogeneity: for the range of $\delta$ values for which heterogeneity improves stability, such probability rapidly approaches one as $n$ grows, while it approaches zero for the other range.

[Figure 5 about here]

For what concerns the threshold for $\delta$ at which heterogeneity turns unstabilising Figure 5 shows ${ }^{3}$ that it decreases with $k$ i.e. the upper bound for $\beta$ and $\gamma$. That both $\delta$ and $k$ should have the same type of effect on the probability of having a stable system (decreasing it as they increase) can be explained as follows: $\delta$ measures, in some sense, the reactivity of the system, while, increasing the value of $k$, the probability of sampling high values for $\beta$ and $\gamma$ also increases which leads to higher forecasts response to small changes in the observed price tampering with the stability of the system. See the Appendix for more on this topic.

[Table 2 about here]

\footnotetext{
${ }^{2}$ In Figure 4 the curve for the $n=1$ cases are the one we analytically derived above in Proposition 2 (for $k=2$ ) and in the appendix (for $k=1$ and 3 ).

${ }^{3}$ Figure 5 depicts the value at which the probability functions when $n=2$ and $n=10$ are equal.
} 
The second set of simulations, whose main findings are reported in Table 2, points out ${ }^{4}$ that stability can emerge in the multi-agent case even if conditions for stability in the representative agent case are not met for every individual. On the other hand stability conditions (resp. violation of) at the individual level for every agent are sufficient to warrant stability (resp. instability), as the first and last row of Table 2 show.

Besides, the following issues can be addressed. Can there be cases in which a stable economy turns unstable if a given group of agents join in? And how about the other way around? Answers are provided in Table 2: its third and fourth rows clearly indicate that one can have mergers of a stable and an unstable group of agents that result either in a stable outcome or an unstable one, with the latter case slightly more frequent. ${ }^{5}$

Our model does not provide any performance-based selection mechanism of intermediaries; this implies that middlemen have, on average, the same abilities and the same behaviour of a single investor. Nonetheless our model shows that, even in this case, a role for middlemen, which has been questioned in several papers, can be justified in that they directly affect market heterogeneity and, in some circumstances, market stability.

Finally, the kind of comparative static approach pursued here could profit a great deal from the introduction of some kind of sensible endogenous mechanism to determine the number of middlemen, which would make the degree of heterogeneity in the market the result of individual decisions, adding complexity and further dimensions to the problems tackled here: this task must be left for future efforts.

\section{References}

[1] Beja, A. \& Goldman, M.B. (1980) On the dynamic behavior of prices in disequilibrium. Journal of Finance, 35, 235-248.

[2] Benassy-Quére, A., Larribeau, S. \& MacDonald, R. (2003). Models of exchange rate expectations: how much heterogeneity? Journal of International Financial Markets, Institutions \& Money, 13, 113-136.

[3] Biglaiser, G. (1993). Middlemen as experts. RAND Journal of Economics, 24(2), 212-223.

[4] Branch, W. (2004). The Theory of Rationally Heterogeneous Expectations: Evidence from Survey Data on Inflation Expectations. The Economic Journal, 114, 592-621.

\footnotetext{
${ }^{4}$ In Table 2 the first column shows labels for the possible outcomes of the mergers: two stable groups that result in an unstable group ("ssu"), or a stable one ("sss"), a stable group and an unstable one that result in a stable group ("sus") or an unstable one ("suu") and two unstable groups that result in an unstable group ("ssu") or a stable one ("uus"). The box on the right displays the number of cases of the "sus" and "suu" categories in which the outcome was determined by a minority of agents: e.g. when a stable group merges with a smaller unstable group giving rise to an unstable group.

${ }^{5}$ It is interesting that about a quarter of these cases come as the result of an "overturning minority", that is the outcome is actually determined by the group that is smaller in size.
}

\begin{tabular}{|c|c|c|c|}
\hline Outcomes & Cases & Overturning minorities & $\%$ \\
\hline sus & 3677 & 1036 & 28.2 \\
\hline suu & 5214 & 1348 & 25.8 \\
\hline
\end{tabular}

Intuitively, this can happen when the larger (say, stable) group is not very robust to injection of new agents if they are individually very far from stability. 
[5] Brock, W., Hommes, C. \& Wagener, F. (2005). Evolutionary dynamics in markets with many trader types. Journal of Mathematical Economics, 41, 7-42.

[6] Colucci, D. \& Valori, V. (2006) Ways of learning in a simple economic setting: a comparative approach. Chaos, Soliton \& Fractals, 29, 653-670.

[7] Day, R.H. \& Huang, W. (1990). Bulls, bears and market sheep. Journal of economic behavior and organization, 14, 299-329.

[8] Egenter, E., Lux, T. \& Stauffer, D. (1999). Finite-size effects in Monte Carlo simulations of two stock market models. Physica A, 268, 250-256.

[9] Elliott, G. \& Ito, T. (1999). Heterogeneous Expectations and Tests of Efficiency in the Yen/Dollar Forward Exchange Rate Market. Journal of Monetary Economics, 43(2), 435-56.

[10] Fingleton, J. (1997). Competition among middlemen when buyers and sellers can trade directly. Journal of Industrial Economics, 45(4), 405-427

[11] Frankel, J.A. \& Froot, K.A. (1990). The Rationality of the Foreign Exchange Rate. Chartists, Fundamentalists and Trading in the Foreign Exchange Market, American Economic Review 80(2), AEA Papers and Proceedings, 181-185.

[12] Hommes, C., Sonnemans, J., Tuinstra J., \& van de Velden, H. (2005). Coordination of Expectations in Asset Pricing Experiments. Review of Financial Studies 18(3), 955-980.

[13] Morris, S. \& Shin, H. (2006). Inertia of Forward-Looking Expectations. American Economic Review, AEA Papers and Proceedings 96(2), 152-157.

[14] Johri, A. \& Leach, J. (2002). Middlemen and the allocation of heterogeneous goods. International Economic Review, 43(2), 347-361.

[15] Rust, J. \& Hal, G. (2003). Middlemen versus market makers: a theory of competitive excange. Journal of Political Economy, 111(2), 353-403.

[16] Vissing-Jorgensen, A. (2002). Towards an Explanation of Household Portfolio Choice Heterogeneity: Nonfinancial Income and Participation Cost Structures. NBER Working Papers 8884. 


\begin{tabular}{|c|c|}
\hline \multicolumn{2}{|c|}{ Values for $n: 2,3,5,10,100$} \\
\hline \multicolumn{2}{|c|}{ Values for $\delta: 0$ to 8 , with a 0.1 step } \\
\hline \multicolumn{2}{|c|}{$\alpha^{(i)}$ from uniform distr. over $[0,1]$} \\
\hline \multicolumn{2}{|c|}{$\beta^{(i)}, \gamma^{(i)}$ from uniform distr. over $[0, k]$, for $k=1,2,3$} \\
\hline Values of $n$ & Number of throws \\
\hline $2,3,5,10$ & 200000 \\
\hline 100 & 20000 \\
\hline
\end{tabular}

Table 1: Simulation's parameters

\begin{tabular}{|l|l|l|}
\hline Outcomes & Nr. of cases & Percentages \\
\hline ssu & 0 & 0 \\
\hline sss & 22146 & $44,29 \%$ \\
\hline sus & 3677 & $7,35 \%$ \\
\hline suu & 5214 & $10,43 \%$ \\
\hline uuu & 18963 & $37,93 \%$ \\
\hline uus & 0 & 0 \\
\hline Total & 50000 & \\
\hline
\end{tabular}

Table 2: Outcomes when two economies are merged

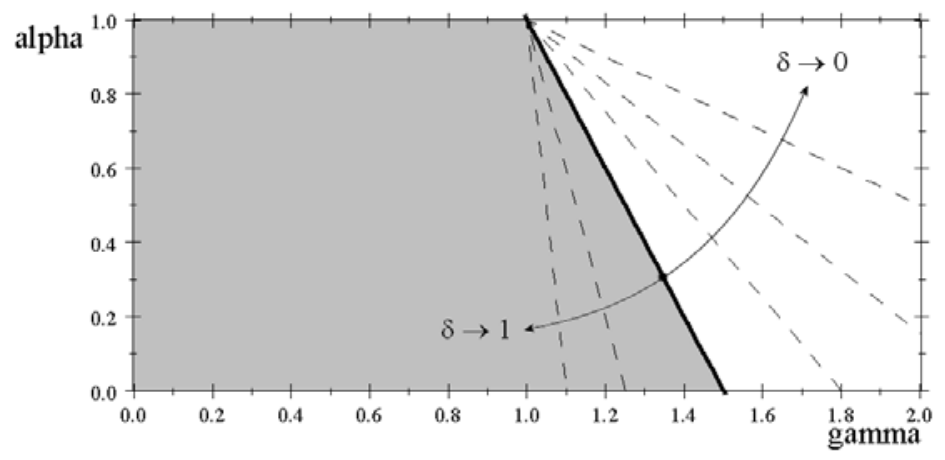

Figure 1: Bifurcation diagram as $\delta$ varies in $(0,1), \beta=\frac{5}{4}$ 


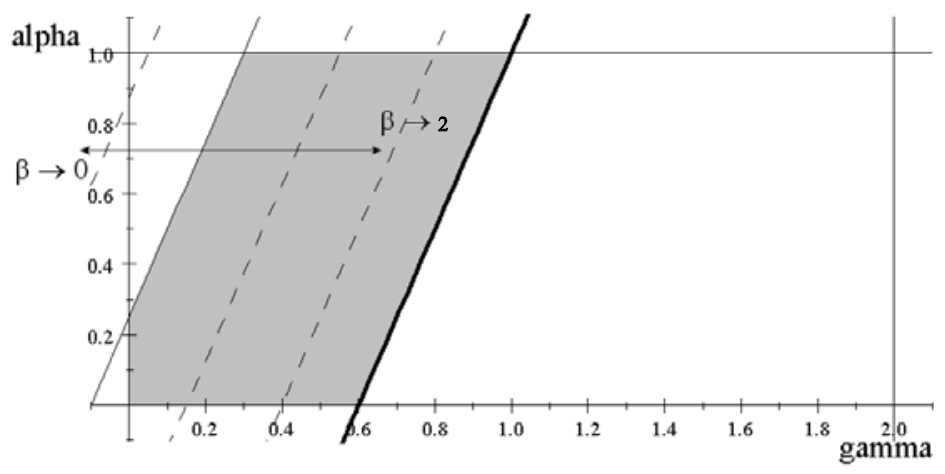

Figure 2: Bifurcation diagram as $\beta$ varies in $(0,2), \delta=\frac{5}{3}$

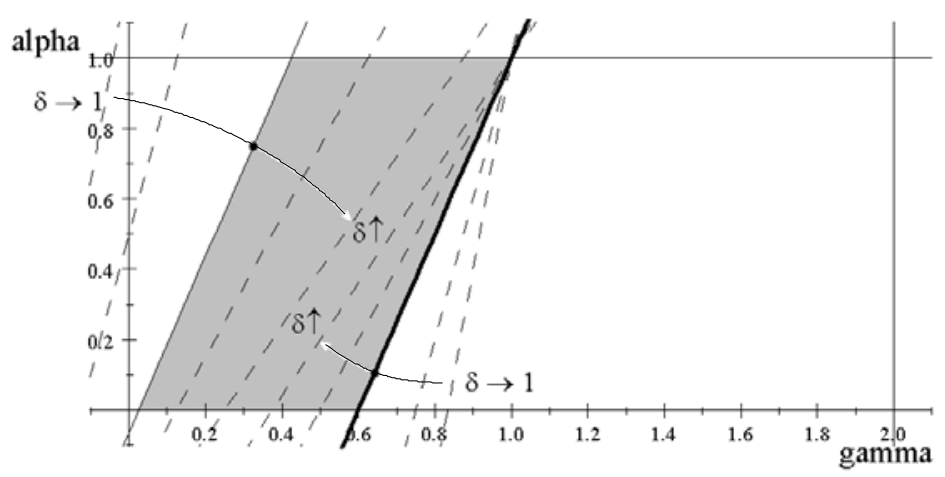

Figure 3: Bifurcation diagram for $\delta>1, \beta=\frac{5}{4}$ 

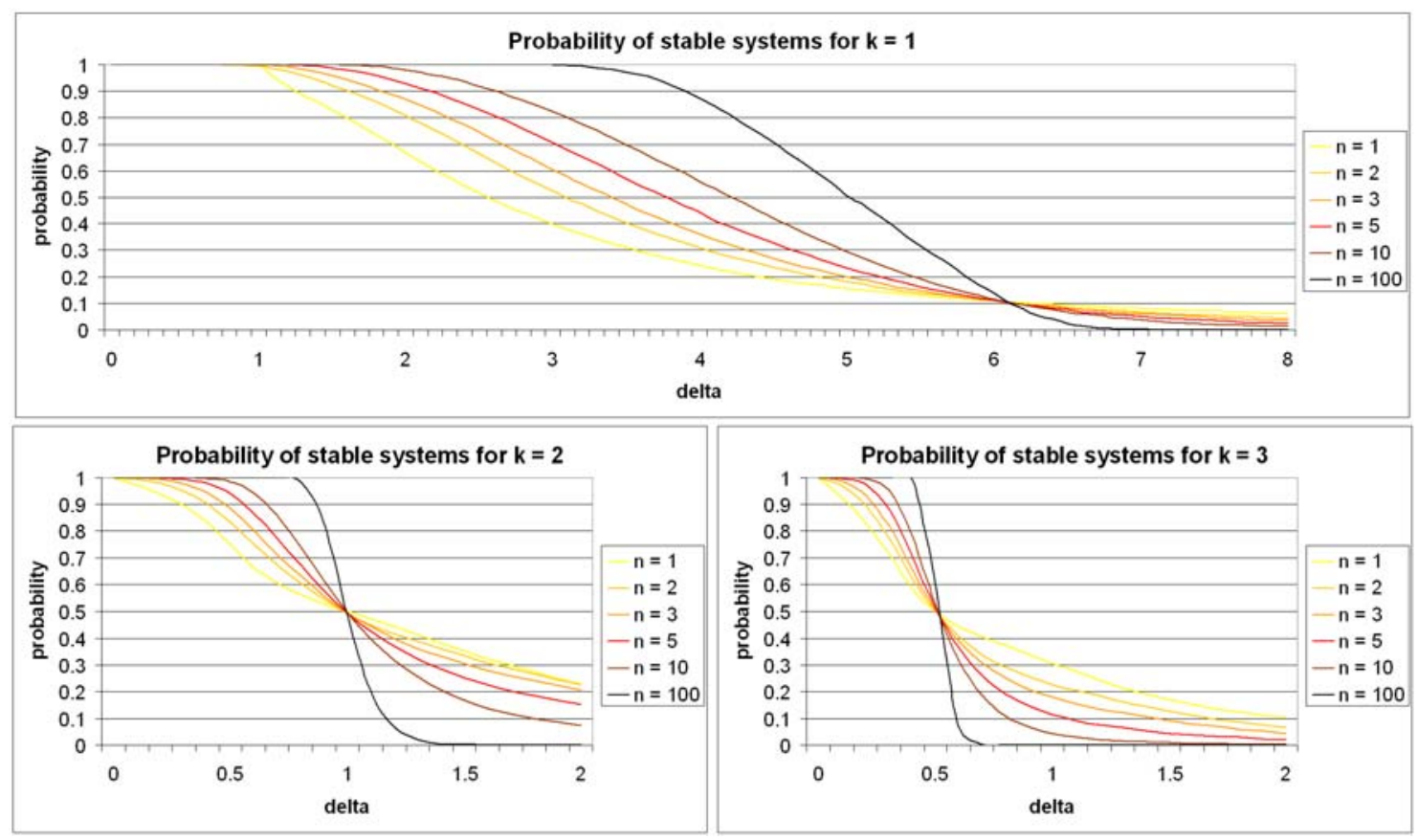

Figure 4: Probability of stable systems as function of $\delta$ (aggregate sensitivity), for various $n$ (measure of heterogeneity)

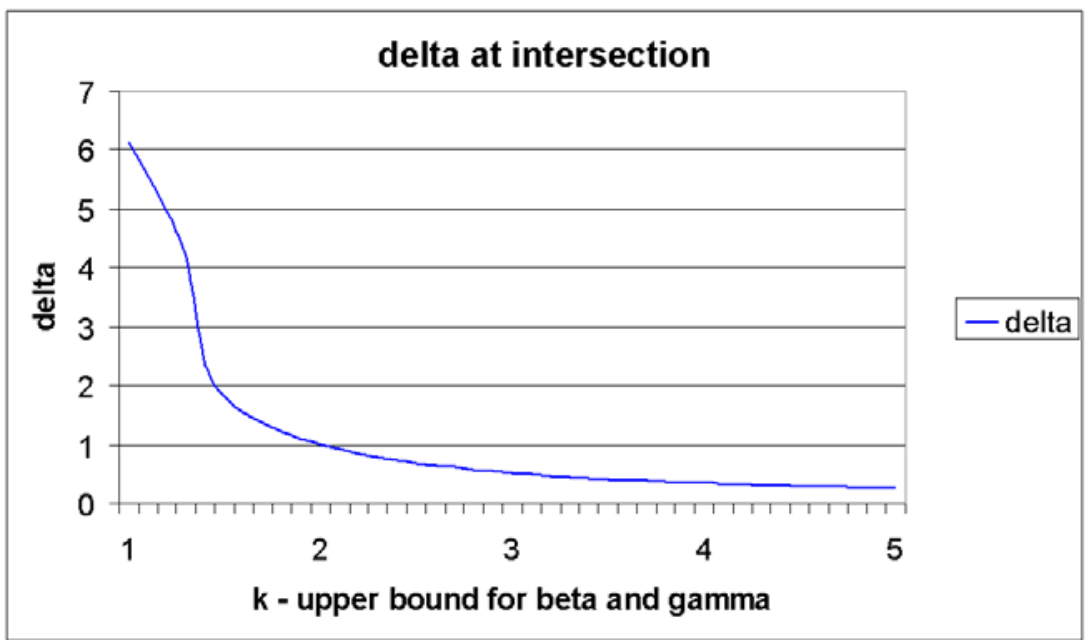

Figure 5: Treshold value for $\delta$ as upper bound for $\beta, \gamma$ varies. 


\section{Appendix}

The probability function in Proposition 2 in the paper stems from the assumption that parameters $\beta$ and $\gamma$ belong to the interval [0,2]. In this appendix we consider the upper bound of such interval as a new parameter and derive the probability of having a stable system supposing that a single mediator is drawn from a uniform distribution on $[0,1] \times[0, k] \times[0, k]$.

\section{The integral}

Remember that the stability conditions of the unique steady state, $\left(p^{*}, p^{e *}, x^{*}\right)=(v, v, v)$, of the system

$$
\left\{\begin{array}{l}
p_{t+1}=p_{t}+\delta\left(\alpha p_{t}^{e}+(1-\alpha) p_{t}-\beta\left(\left(p_{t}-v\right)+\left(p_{t}-v\right)^{3}\right)+\gamma\left(p_{t}-x_{t}\right)-p_{t}\right) \\
p_{t+1}^{e}=\alpha p_{t}^{e}+(1-\alpha) p_{t}-\beta\left(\left(p_{t}-v\right)+\left(p_{t}-v\right)^{3}\right)+\gamma\left(p_{t}-x_{t}\right) \\
x_{t+1}=p_{t}
\end{array}\right.
$$

are

$$
\begin{aligned}
& \frac{\delta\left(\gamma-\frac{\beta}{2}\right)+1}{\delta-1}<\alpha<\frac{\gamma \delta-1}{\delta-1} \text { if } \delta<1 \\
& \frac{\beta}{2}-1<\gamma<1 \text { if } \delta=1 \\
& \frac{\gamma \delta-1}{\delta-1}<\alpha<\frac{\delta\left(\gamma-\frac{\beta}{2}\right)+1}{\delta-1} \text { if } \delta>1
\end{aligned}
$$

which can be equivalently written as

$$
\begin{cases}\beta<\frac{2+2 \delta \gamma-2 \alpha(\delta-1)}{\delta} \triangleq P D_{\delta}(\gamma, \alpha) & \text { if } \delta \neq 1 \\ \gamma<\frac{1+\alpha(\delta-1)}{\delta} \triangleq N S_{\delta}(\alpha) & \text { if } \delta=1 \\ \beta<2 \gamma+2 & \end{cases}
$$

Supposing that the parameters $(\alpha, \beta, \gamma)$ are drawn from a uniform distribution on $[0,1] \times[0, k] \times$ $[0, k]$, the probability of having a stable system for a given couple of parameters $(\delta, k)$ can be written as

$$
P(\delta, k)=\frac{1}{k^{2}} \int_{0}^{1} \int_{0}^{\max \left\{0, \min \left\{N S_{\delta}(\alpha), k\right\}\right\}} \max \left\{0, \min \left\{P D_{\delta}(\gamma, \alpha), k\right\}\right\} d \gamma d \alpha
$$

\section{Expliciting the $\max -\min$ functions}

In order to evaluate the integral, it is relevant to understand when $P D_{\delta}(\gamma, \alpha)$ and $N S_{\delta}(\alpha)$ belong to $[0, k]$, given the restrictions on their arguments. The following figure shows the function $\gamma=N S_{\delta}(\alpha)$ for $\delta=\{0.5$ to 3 step 0.5$\}$ (darkness decreases with $\delta$ ) 


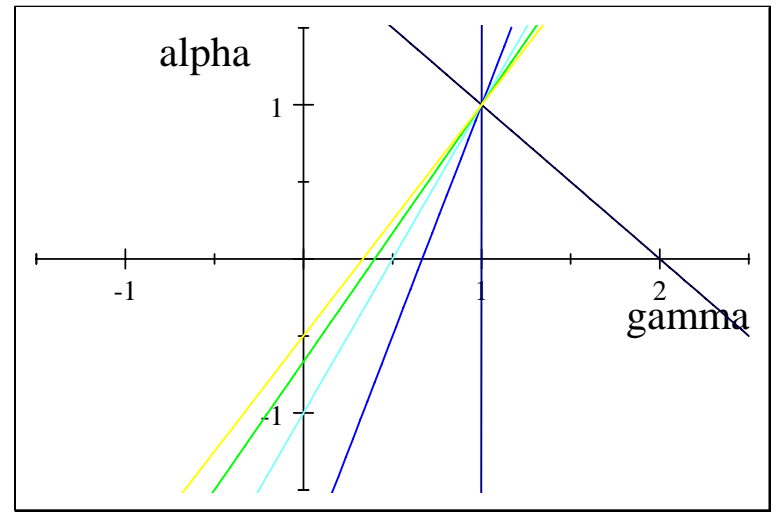

The function $\gamma=N S_{\delta}(\alpha)$ is a bundle of lines centred in $(1,1)$ and, for $\delta>0$, always intersecting the positive half of the $\gamma-$ axis (in $\gamma=\frac{1}{\delta}$ ). So $N S_{\delta}(\alpha)>0$ is always satisfied whereas $N S_{\delta}(\alpha)<k$ holds

$$
\begin{aligned}
& \text { for all } \alpha \in[0,1] \text { if } k>\max \left\{1, \frac{1}{\delta}\right\} \\
& \text { for } \frac{1-k \delta}{1-\delta}<\alpha \leq 1 \text { if } \delta \leq 1 \text { and } 1<k<\frac{1}{\delta} \\
& \text { for } 0<\alpha \leq \frac{k \delta-1}{\delta-1} \text { if } \delta>1 \text { and } \frac{1}{\delta}<k<1 \\
& \text { never if } k<\min \left\{1, \frac{1}{\delta}\right\}
\end{aligned}
$$

Now consider $\beta=P D_{\delta}(\gamma, \alpha)$. The following figure shows $P D_{\delta}(\gamma, \alpha)=0$ for $\delta=\{0.5$ to 3 step 0.5$\}$ (darkness decreases with $\delta$ )

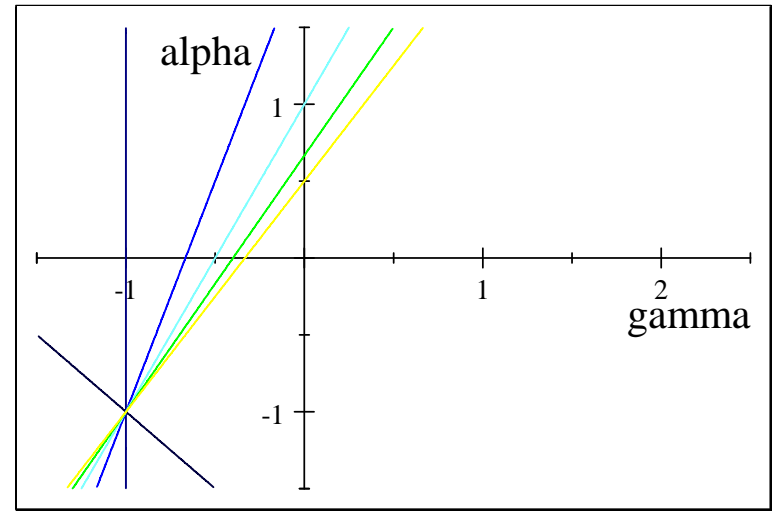

Qualitatively, increasing $\delta$, the slope of the line decreases and tends to 1 . From $P D_{\delta}(\gamma, \alpha)=0$, we obtain

$$
\alpha=\frac{\delta}{\delta-1} \gamma+\frac{1}{\delta-1} \triangleq h_{\delta}(\gamma)
$$


As $h_{\delta}(0)=\frac{1}{\delta-1}$ belongs to $(0,1)$ if and only if $\delta>2$, condition $P D_{\delta}(\gamma, \alpha)>0$ is always satisfied whenever $\delta \leq 2$. Otherwise, when $\delta>2, P D_{\delta}(\gamma, \alpha)>0$ holds

$$
\begin{aligned}
& \text { for all } \alpha \in[0,1] \text { if } \gamma \geq \frac{\delta-2}{\delta} \\
& \text { for } \alpha \leq \frac{\delta \gamma+1}{\delta-1} \text { if } \gamma<\frac{\delta-2}{\delta}
\end{aligned}
$$

Further, from equation $P D_{\delta}(\gamma, \alpha)=k$ we obtain

$$
\alpha=\frac{\delta}{\delta-1} \gamma+\frac{2-\delta k}{2(\delta-1)} \triangleq f_{\delta, k}(\gamma)
$$

From the fact that $f_{\delta, k}(\gamma)=1$ when $\gamma=\frac{2 \delta+k \delta-4}{2 \delta}$, that $f_{\delta, k}(\gamma)=0$ when $\gamma=\frac{k \delta-2}{2 \delta}$ and that $f_{\delta, k}(0)=\frac{2-\delta k}{2(\delta-1)}$, follows that $P D_{\delta}(\gamma, \alpha)<k$ holds: when $\delta>1$

$$
\begin{aligned}
& \text { for all } \alpha \in[0,1] \text { if } \gamma \leq \frac{k \delta-2}{2 \delta} \text { (interesting when } k>\frac{2}{\delta} \text { ) } \\
& \text { for } \alpha>\frac{2 \delta \gamma+2-\delta k}{2(\delta-1)} \text { if } \gamma \leq \frac{2 \delta+k \delta-4}{2 \delta} \text { (interesting when } k>\frac{4-2 \delta}{\delta} \text { ) } \\
& \text { never if } k \leq \frac{4-2 \delta}{\delta} \text { or if } \gamma>\frac{2 \delta+k \delta-4}{2 \delta} \text { (interesting when } k<\frac{4}{\delta} \text { ) }
\end{aligned}
$$

and when $\delta<1$

$$
\begin{aligned}
& \text { for all } \alpha \in[0,1] \text { if } \gamma \leq \frac{2 \delta+k \delta-4}{2 \delta} \text { (interesting when } k>\frac{4-2 \delta}{\delta} \text { ) } \\
& \text { for } \alpha<\frac{2 \delta \gamma+2-\delta k}{2(\delta-1)} \text { if } \gamma \leq \frac{k \delta-2}{2 \delta} \text { (interesting when } k>\frac{2}{\delta} \text { ) } \\
& \text { never if } k \leq \frac{2}{\delta} \text { or if } \gamma>\frac{k \delta-2}{2 \delta} \text { (interesting when } k<\frac{4}{\delta} \text { ) }
\end{aligned}
$$

Finally, observing that $k=\frac{2 \delta+k \delta-4}{2 \delta}$ implies $k=\frac{2 \delta-4}{\delta}$, and putting everything together, we have that, in order to evaluate the integral, the following set of values for $k$ is relevant:

$$
\left\{\frac{\delta-2}{\delta}, \frac{2 \delta-4}{\delta}, 1, \frac{1}{\delta}, \frac{2}{\delta}, \frac{4-2 \delta}{\delta}, \frac{4}{\delta}\right\}
$$

when $\delta \neq 1$ and 
when $\delta=1$. We will, then, distinguish among 10 different cases for $\delta$ which are summarized in the following table:

\begin{tabular}{|c|c|c|}
\hline 1 & $\overline{\delta<1}$ & 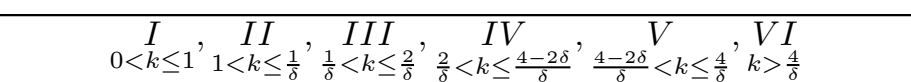 \\
\hline 2 & $\delta=1$ & $\underset{0<k<1}{I}, \underset{1<k<2}{V I I}, \underset{2<k<4}{V I I I}, \underset{k>4}{I X}$ \\
\hline 3 & $1<\delta \leq \frac{4}{3}$ & $\underset{0<k \leq \frac{1}{\delta}}{I}, \underset{\frac{1}{\delta}<k \leq 1}{X}, \underset{1<k \leq \frac{4-2 \delta}{\delta},}{I I I}, \underset{\frac{4-2 \delta}{\delta}<<\leq \frac{2}{\delta}}{X}, \underset{\frac{2}{\delta}<k \leq \frac{4}{\delta}}{V}, \underset{k>\frac{4}{\delta}}{V I}$ \\
\hline 4 & $\frac{4}{3}<\delta \leq \frac{3}{2}$ & $\underset{0<k \leq \frac{1}{\delta}}{I}, \underset{\frac{1}{\delta}<k \leq \frac{4-2 \delta}{\delta}}{X}, \underset{\frac{4-2 \delta}{\delta}<k \leq 1}{X I I}, \underset{1<k \leq \frac{2}{\delta}}{X I}, \underset{\frac{2}{\delta}<k \leq \frac{4}{\delta}}{V}, \underset{k>\frac{4}{\delta}}{V I}$ \\
\hline 5 & $\frac{3}{2}<\delta \leq 2$ & $\underset{0<k \leq \frac{4-2 \delta}{\delta},}{I}, \underset{\frac{4-2 \delta}{\delta}<k \leq \frac{1}{\delta}}{X I I I}, \underset{\frac{1}{\delta}<k \leq 1}{X I I}, \underset{1<k \leq \frac{2}{\delta}}{X I}, \underset{\frac{2}{\delta}<k \leq \frac{4}{\delta}}{V}, \underset{k>\frac{4}{\delta}}{V I}$ \\
\hline 6 & $2<\delta \leq \frac{5}{2}$ & 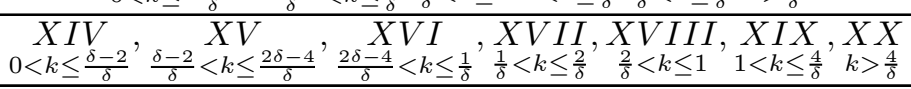 \\
\hline 7 & $\frac{5}{2}<\delta \leq 3$ & 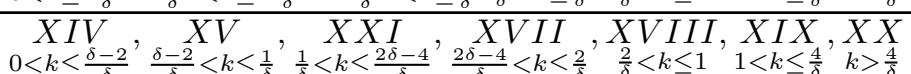 \\
\hline 8 & $3<\delta \leq 4$ & $\underset{0<k \leq \frac{1}{\delta}}{X I V}, \underset{\frac{1}{\delta}<k \leq \frac{\delta-2}{\delta}}{X X I I}, \underset{\frac{\delta-2}{\delta}<X I \leq \frac{2}{\delta}}{X X}, \underset{\frac{2}{\delta}<k \leq \frac{2 \delta-4}{\delta}}{X X I I I}, \underset{\frac{2 \delta-4}{\delta}<V k \leq 1}{X V I I}, \underset{1<k \leq \frac{4}{\delta}}{X I X}, \underset{k>\frac{4}{\delta}}{X X}$ \\
\hline 9 & $4<\delta \leq 6$ & 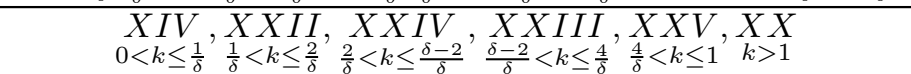 \\
\hline 10 & $\delta>6$ & $\underset{0<k \leq \frac{1}{\delta}}{X I V}, \underset{\frac{1}{\delta}<k \leq \frac{2}{\delta}}{X X I I}, \underset{\frac{2}{\delta}<k \leq \frac{4}{\delta}}{X X I V}, \underset{\frac{4}{\delta}<k \leq 1}{X X}, \underset{k>1}{X X}$ \\
\hline
\end{tabular}

The table should be read as follows: given a couple $(\delta, k)$ one needs to find the appropriate row in column 2 depending on $\delta$ and then column 3 gives the appropriate function ( $I$ to $X X V$ ) depending on $k$. Functions $I$ to $X X V$ are derived below. For example, for $\delta=1.1$ and $k=2$ row 3 is relevant and $\frac{2}{\delta} \leq k \leq \frac{4}{\delta}$ applies so the probability is given by function $V$ (see below), that is

$$
\left.\frac{-3 k^{2} \delta^{2}+24 k \delta-4 \delta^{2}+20 \delta-28}{12 k^{2} \delta^{2}}\right|_{\delta=1.1, k=2} \sim 0.47
$$

\section{Functions $I$ to $X X V$}

We now derive every single part of the probability function. Every sub-section of the integral will be introduced by a group of graphs referring to the different sub-cases to which the integral applies. Each graph shows one or more of the lines $N S_{\delta}(\alpha)$ (blue), $P D_{\delta}(\gamma, \alpha)=k$ (red) and $P D_{\delta}(\gamma, \alpha)=0$ (green) and evidences in light grey the region in which the function to be integrated is $P D_{\delta}(\gamma, \alpha)$ and in dark grey the region in which the function to be integrated is the constant $k$.

Case I
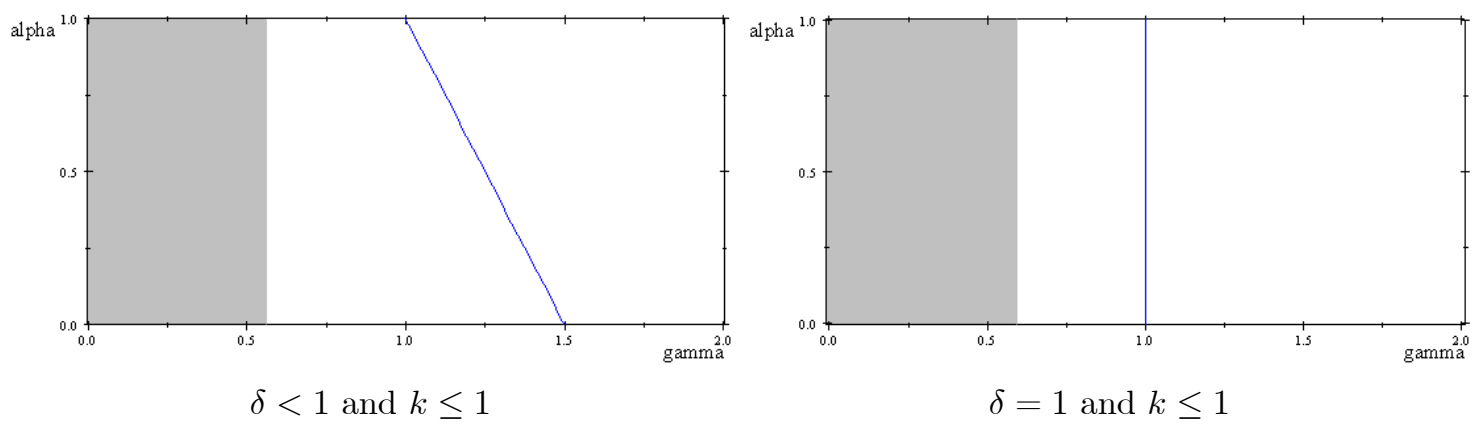


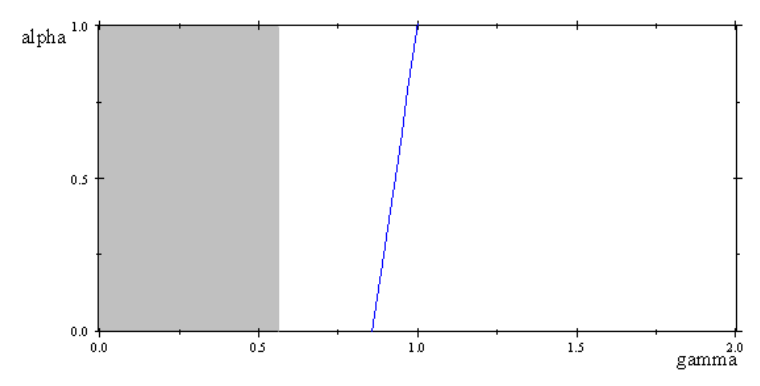

$1<\delta \leq \frac{4}{3}$ and $k \leq \frac{1}{\delta}$

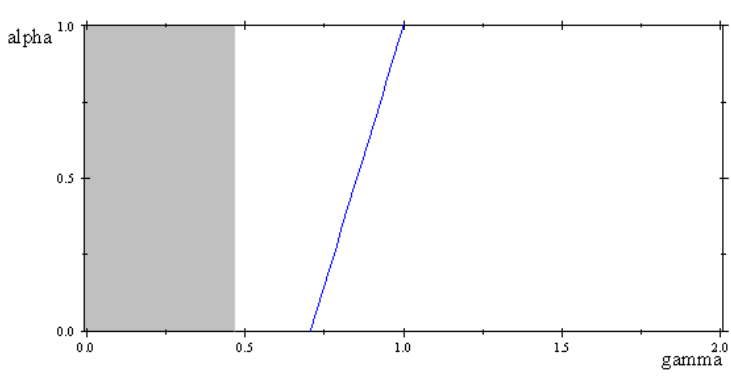

$\frac{4}{3}<\delta \leq \frac{3}{2}$ and $k \leq \frac{1}{\delta}$

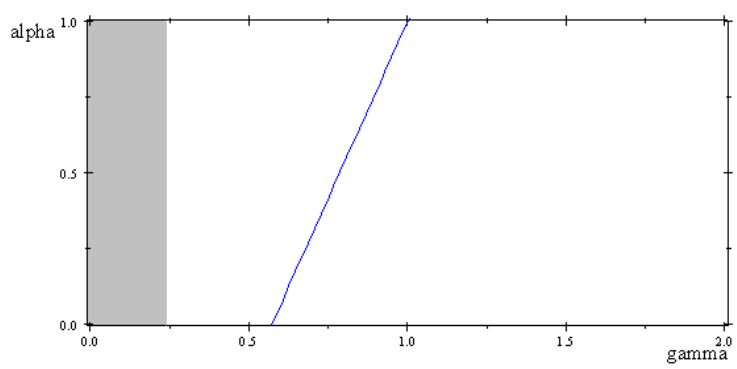

$\frac{3}{2}<\delta \leq 2$ and $k \leq \frac{4-2 \delta}{\delta}$

$$
\frac{1}{k^{2}} \int_{0}^{k} \int_{0}^{1} k d \alpha d \gamma=1
$$

\section{Case II}

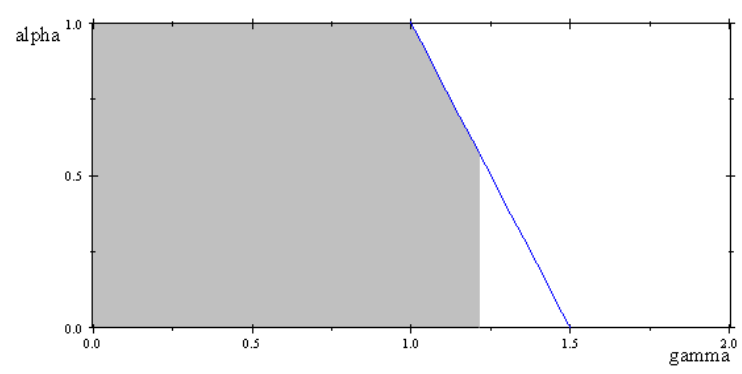

$\delta<1$ and $1<k<\frac{1}{\delta}$

$$
\frac{1}{k^{2}}\left(\int_{0}^{1} \int_{0}^{1} k d \alpha d \gamma+\int_{1}^{k} \int_{0}^{\frac{1-\gamma \delta}{1-\delta}} k d \alpha d \gamma\right)=\frac{2 k-\delta-\delta k^{2}}{2 k(1-\delta)}
$$

\section{Case III}
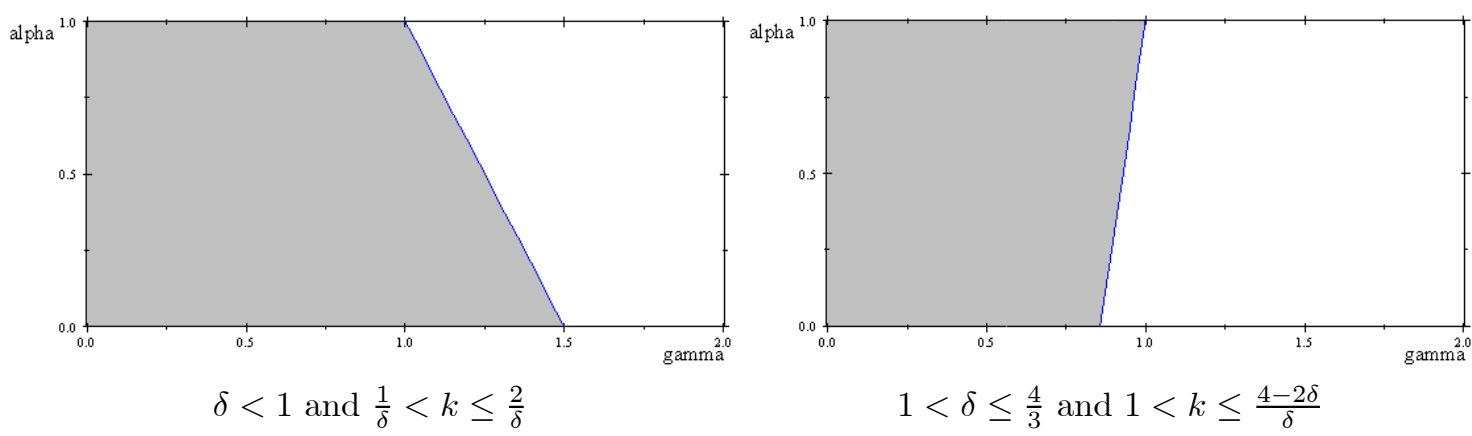


\section{Case IV}

$$
\frac{1}{k^{2}} \int_{0}^{1} \int_{0}^{\frac{1-\alpha+\alpha \delta}{\delta}} k d \gamma d \alpha=\frac{\delta+1}{2 k \delta}
$$

$$
\begin{aligned}
& \frac{1}{k^{2}}\left(\int_{0}^{\frac{\delta k-2}{2 \delta}} \int_{0}^{\frac{\delta}{\delta-1} \gamma+\frac{2-\delta k}{2(\delta-1)}} P D(\gamma, \alpha, \delta) d \alpha d \gamma+\int_{0}^{\frac{\delta k-2}{2 \delta}} \int_{\frac{\delta}{\delta-1} \gamma+\frac{2-\delta k}{2(\delta-1)}}^{1} k d \alpha d \gamma+\int_{0}^{1} \int_{\frac{\delta k-2}{2 \delta}}^{\frac{1-\alpha+\alpha \delta}{\delta}} k d \gamma d \alpha\right)= \\
& =\frac{-k^{3} \delta^{3}+6 k^{2} \delta^{2}-12 k \delta^{3}+8}{24 k^{2} \delta^{2}(1-\delta)}
\end{aligned}
$$

\section{Case V}
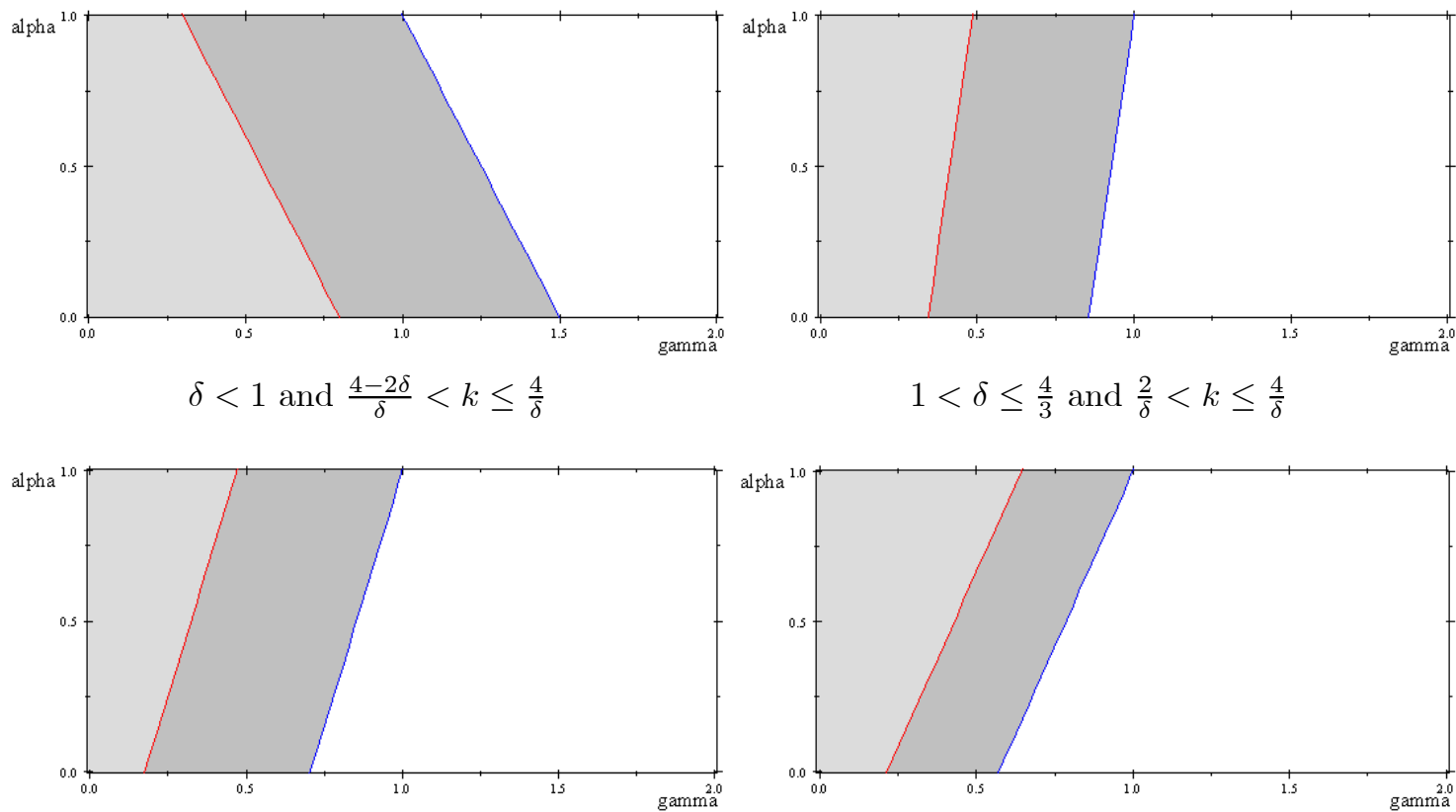

$\frac{4}{3}<\delta \leq \frac{3}{2}$ and $\frac{2}{\delta}<k \leq \frac{4}{\delta}$

$$
\frac{3}{2}<\delta \leq 2 \text { and } \frac{2}{\delta}<k \leq \frac{4}{\delta}
$$

$$
\begin{aligned}
& \frac{1}{k^{2}}\left(\int_{0}^{1} \int_{0}^{\frac{-2 \alpha+k \delta+2 \alpha \delta-2}{2 \delta}} P D(\gamma, \alpha, \delta) d \gamma d \alpha+\int_{0}^{1} \int_{\frac{-2 \alpha+k \delta+2 \alpha \delta-2}{2 \delta}}^{\frac{1-\alpha+\alpha \delta}{\delta}} k d \gamma d \alpha\right)= \\
= & \frac{-3 k^{2} \delta^{2}+24 k \delta-4 \delta^{2}+20 \delta-28}{12 k^{2} \delta^{2}}
\end{aligned}
$$




\section{Case VI}
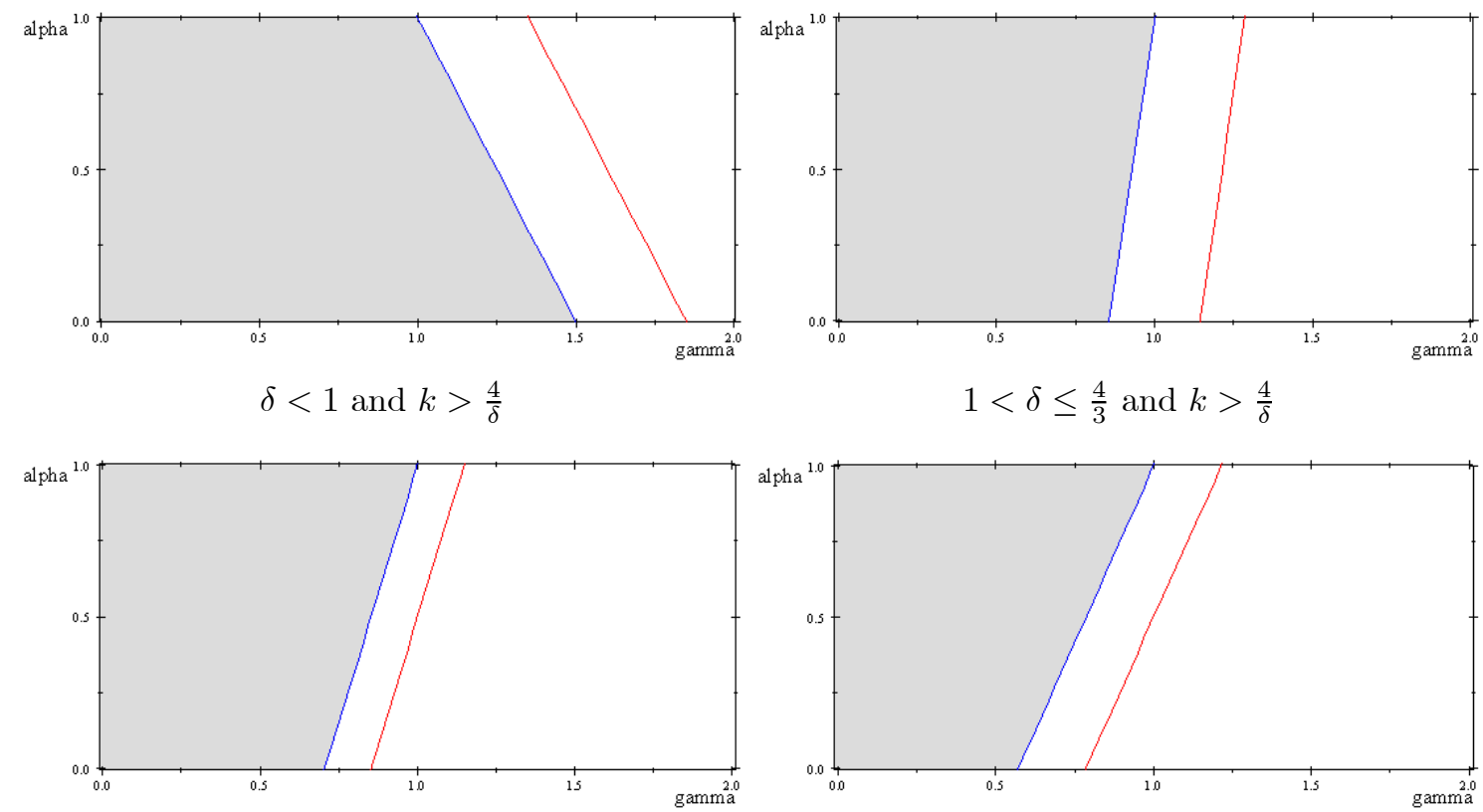

$1<\delta \leq \frac{4}{3}$ and $k>\frac{4}{\delta}$

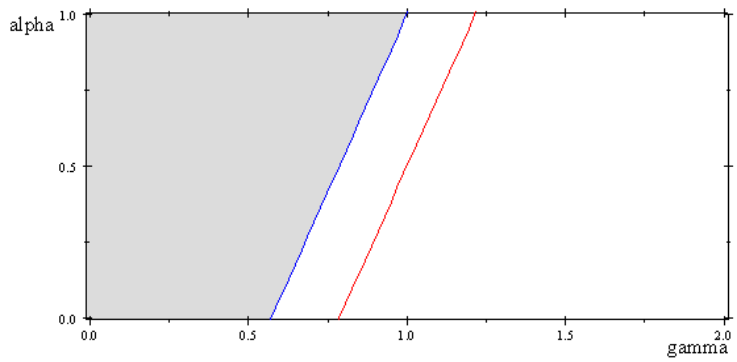

$$
\frac{4}{3}<\delta \leq \frac{3}{2} \text { and } k>\frac{4}{\delta}
$$

$\frac{3}{2}<\delta \leq 2$ and $k>\frac{4}{\delta}$

$$
\frac{1}{k^{2}} \int_{0}^{1} \int_{0}^{\frac{1-\alpha+\alpha \delta}{\delta}} P D(\gamma, \alpha, \delta) d \gamma d \alpha=\frac{-\delta^{2}+5 \delta+5}{3 k^{2} \delta^{2}}
$$

\section{Case VII}

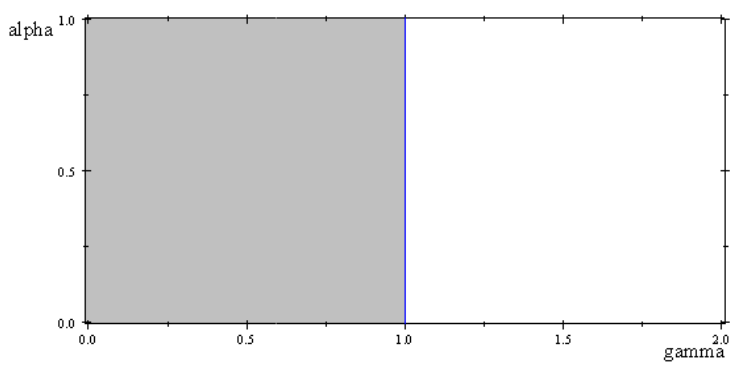

$\delta=1$ and $1<k \leq 2$

$\frac{1}{k^{2}} \int_{0}^{1} \int_{0}^{1} k d \alpha d \gamma=\frac{1}{k}$

\section{Case VIII}

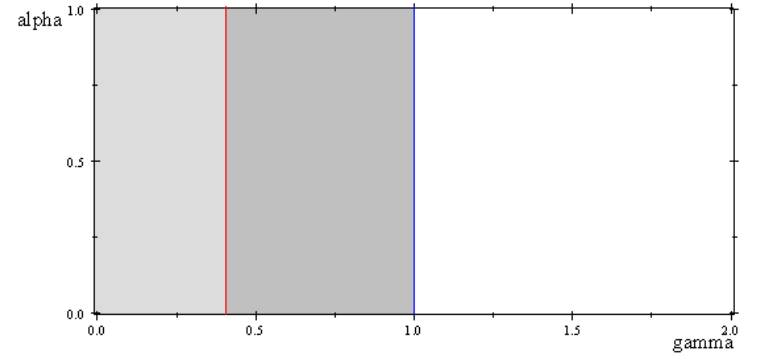

$\delta=1$ and $2<k \leq 4$ 


$$
\frac{1}{k^{2}}\left(\int_{0}^{1} \int_{0}^{\frac{k}{2}-1} D(\gamma, \alpha, 1) d \gamma d \alpha+\int_{0}^{1} \int_{\frac{k}{2}-1}^{1} k d \gamma d \alpha\right)=\frac{-k^{2}+8 k-4}{4 k^{2}}
$$

\section{Case IX}

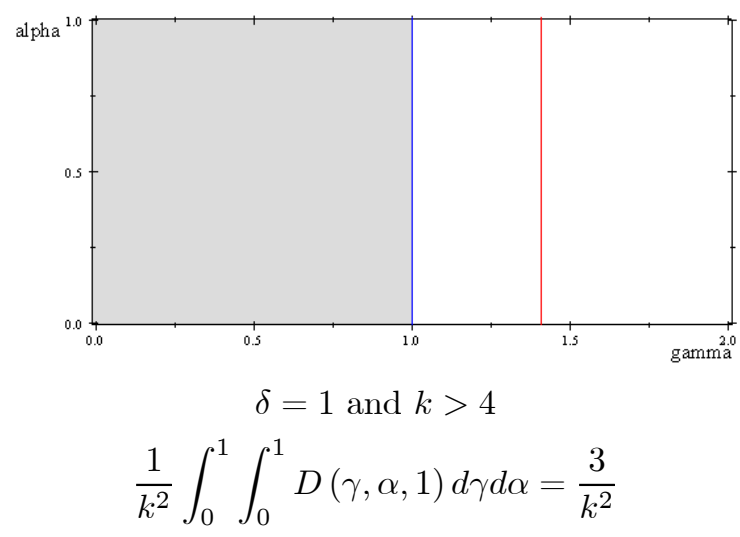

Case X
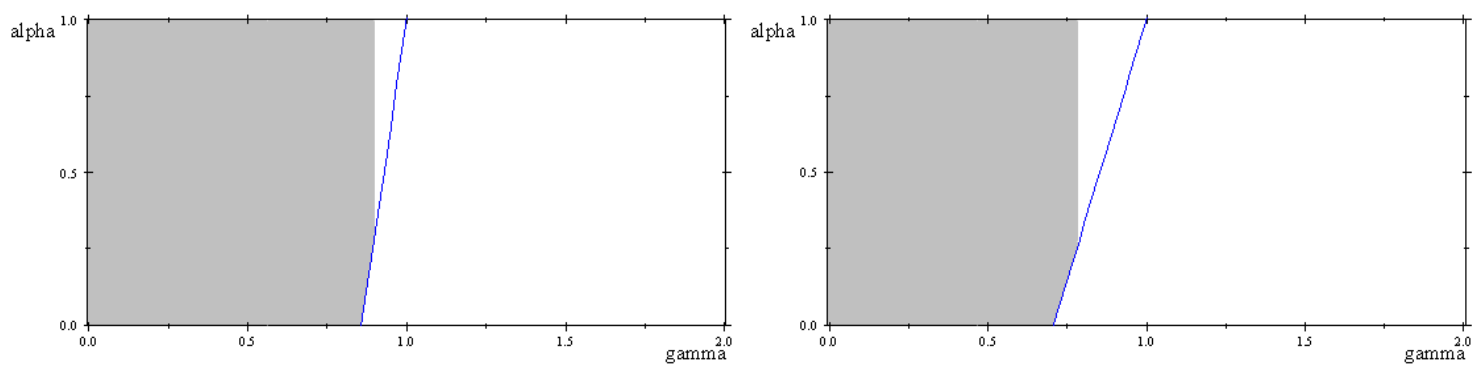

$1<\delta \leq \frac{4}{3}$ and $\frac{1}{\delta}<k \leq 1$

$\frac{4}{3}<\delta \leq \frac{3}{2}$ and $\frac{1}{\delta}<k \leq \frac{4-2 \delta}{\delta}$

$$
\frac{1}{k^{2}}\left(\int_{0}^{\frac{1}{\delta}} \int_{0}^{1} k d \alpha d \gamma+\int_{\frac{1}{\delta}}^{k} \int_{\frac{1-\gamma \delta}{1-\delta}}^{1} k d \alpha d \gamma\right)=\frac{k^{2} \delta^{2}-2 k \delta^{2}+1}{2 k \delta(1-\delta)}
$$

\section{Case XI}

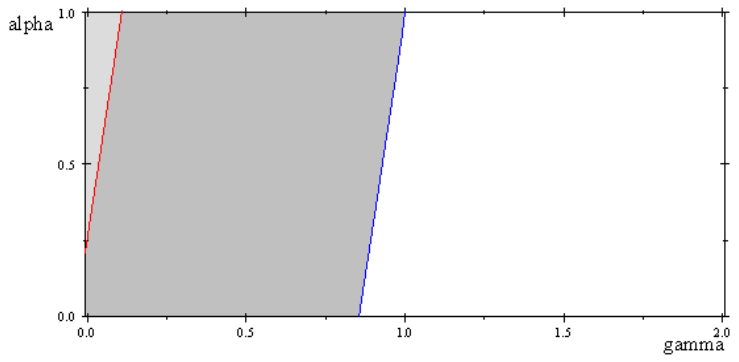

$1<\delta \leq \frac{4}{3}$ and $\frac{4-2 \delta}{\delta}<k \leq \frac{2}{\delta}$

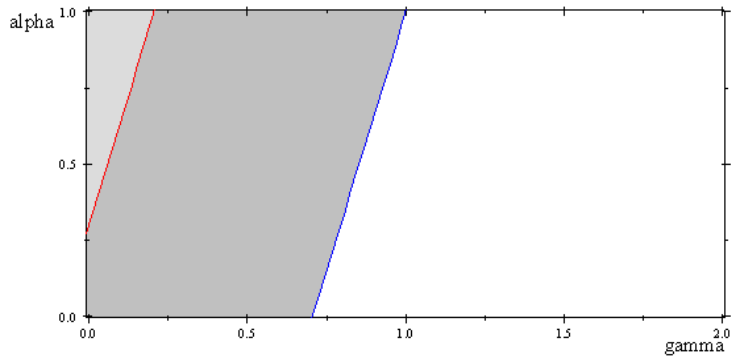

$\frac{4}{3}<\delta \leq \frac{3}{2}$ and $1<k \leq \frac{2}{\delta}$ 


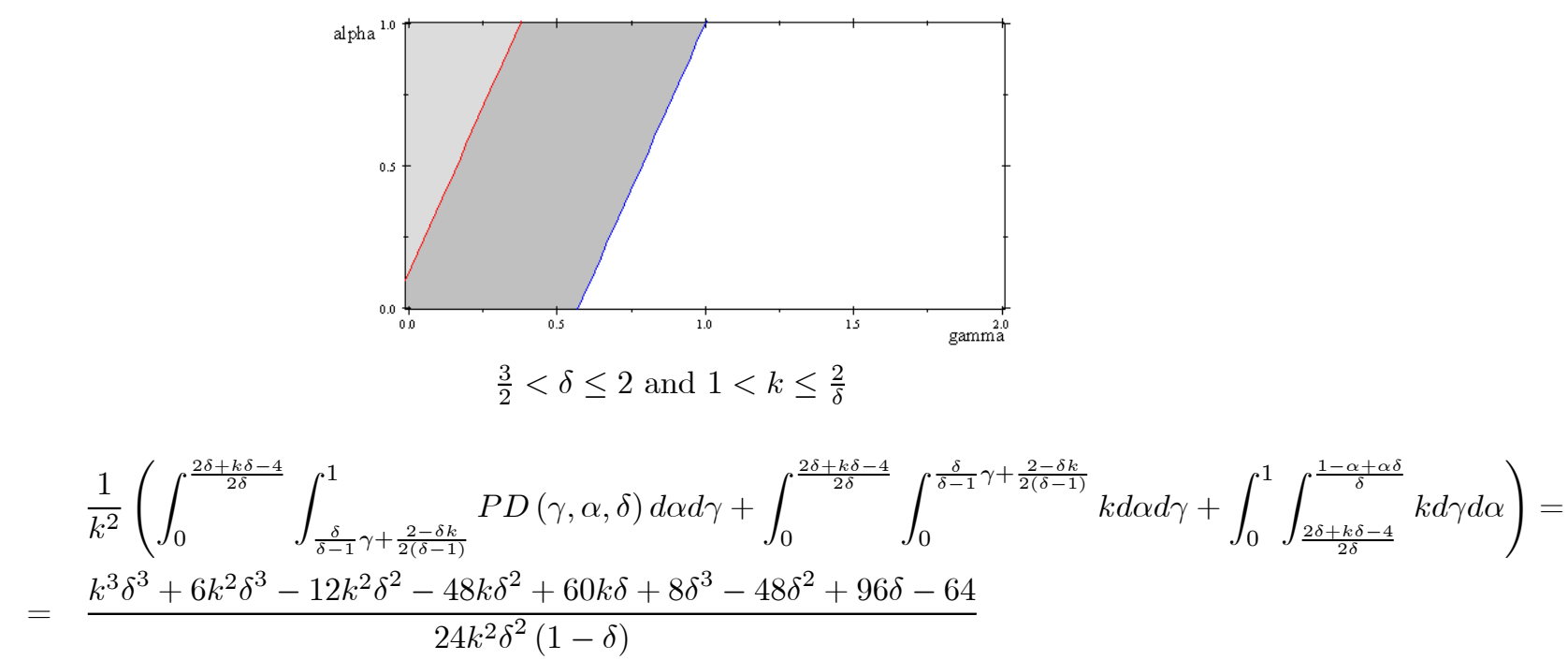

\section{Case XII}

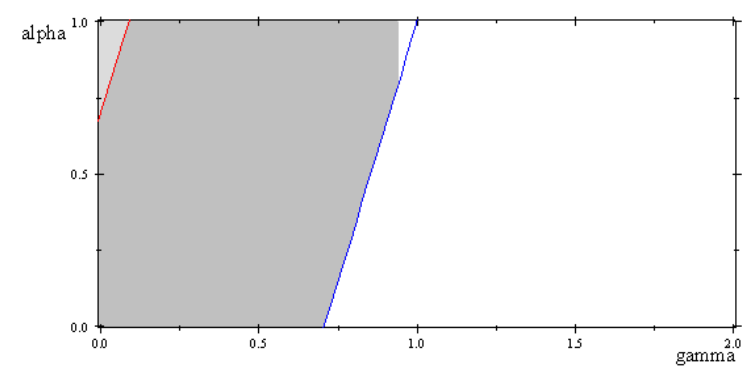

$\frac{4}{3}<\delta \leq \frac{3}{2}$ and $\frac{4-2 \delta}{\delta}<k \leq 1$

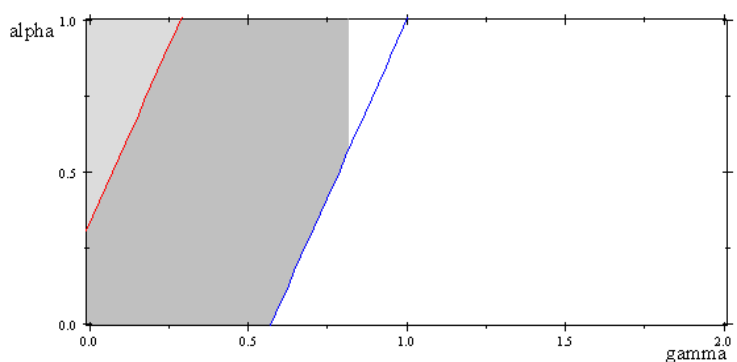

$\frac{3}{2}<\delta \leq 2$ and $\frac{1}{\delta}<k \leq 1$

$\frac{1}{k^{2}} \int_{0}^{\frac{2 \delta+k \delta-4}{2 \delta}} \int_{\frac{\delta}{\delta-1} \gamma+\frac{2-\delta k}{2(\delta-1)}}^{1} P D(\gamma, \alpha, \delta) d \alpha d \gamma+\frac{1}{k^{2}} \int_{0}^{\frac{2 \delta+k \delta-4}{2 \delta}} \int_{0}^{\frac{\delta}{\delta-1} \gamma+\frac{2-\delta k}{2(\delta-1)}} k d \alpha d \gamma+\frac{1}{k^{2}} \int_{0}^{1} \int_{\frac{2 \delta+k \delta-4}{2 \delta}}^{\frac{1}{\delta}} k d \gamma d \alpha+$

$+\quad \frac{1}{k^{2}} \int_{\frac{1}{\delta}}^{k} \int_{\frac{\delta}{\delta-1} \gamma-\frac{1}{\delta-1}}^{1} k d \alpha d \gamma=\frac{13 k^{3} \delta^{3}-18 k^{2} \delta^{3}-12 k^{2} \delta^{2}+12 k \delta^{3}-48 k \delta^{2}+60 k \delta+8 \delta^{3}-48 \delta^{2}+96 \delta-64}{24 k^{2} \delta^{2}(1-\delta)}$

\section{Case XIII}

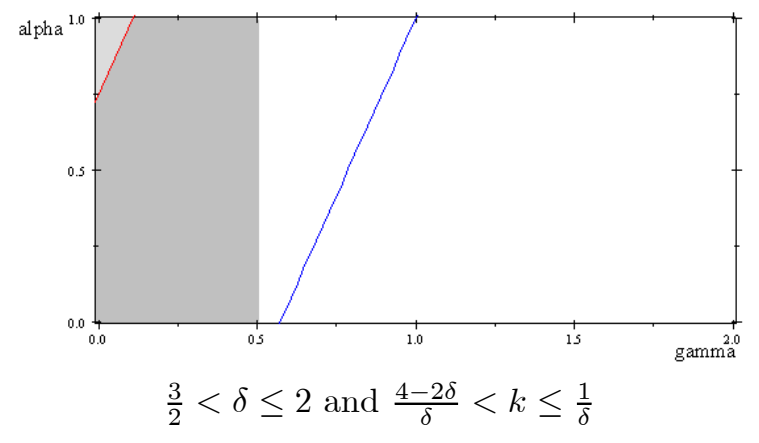




$$
\begin{aligned}
& \frac{1}{k^{2}}\left(\int_{0}^{\frac{2 \delta+k \delta-4}{2 \delta}} \int_{\frac{\delta}{\delta-1} \gamma+\frac{2-\delta k}{2(\delta-1)}}^{1} P D(\gamma, \alpha, \delta) d \alpha d \gamma+\int_{0}^{\frac{2 \delta+k \delta-4}{2 \delta}} \int_{0}^{\frac{\delta}{\delta-1} \gamma+\frac{2-\delta k}{2(\delta-1)}} k d \alpha d \gamma+\int_{0}^{1} \int_{\frac{2 \delta+k \delta-4}{2 \delta}}^{k} k d \gamma d \alpha\right)= \\
= & \frac{k^{3} \delta^{3}-18 k^{2} \delta^{3}+12 k^{2} \delta^{2}+12 k \delta^{3}-48 k \delta^{2}+48 k \delta+8 \delta^{3}-48 \delta^{2}+96 \delta-64}{24 k^{2} \delta^{2}(1-\delta)}
\end{aligned}
$$

\section{Case XIV}

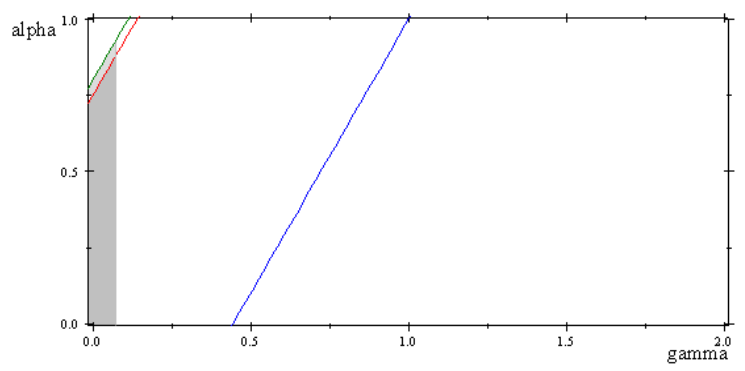

$2<\delta \leq \frac{5}{2}$ and $k \leq \frac{\delta-2}{\delta}$

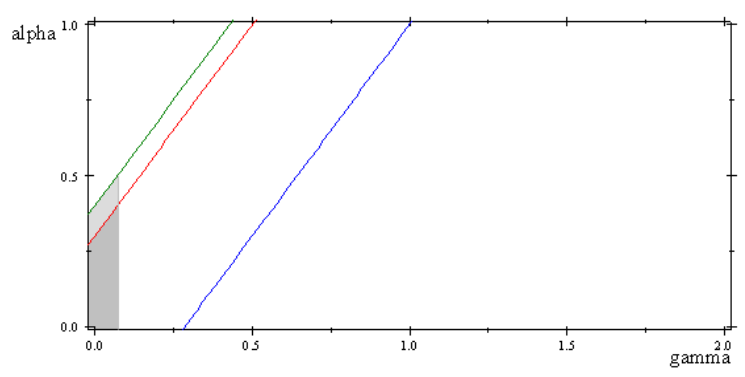

$3<\delta \leq 4$ and $k \leq \frac{1}{\delta}$

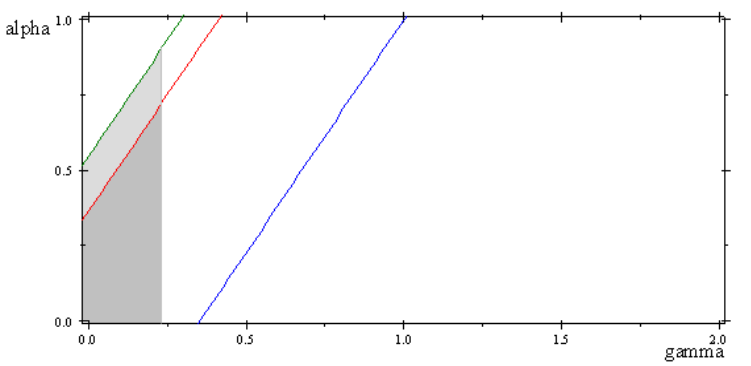

$\frac{5}{2}<\delta \leq 3$ and $k \leq \frac{\delta-2}{\delta}$

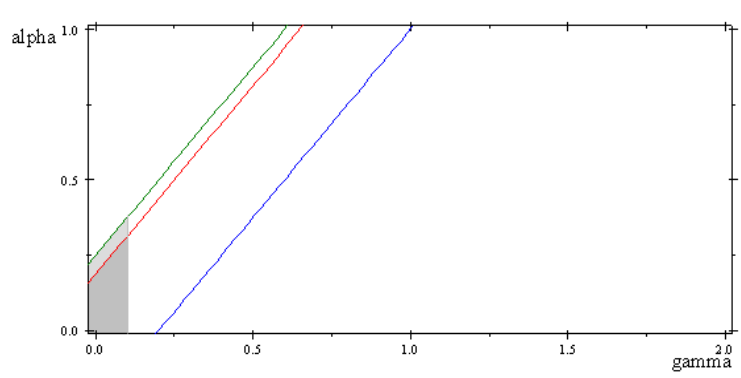

$4<\delta \leq 6$ and $k \leq \frac{1}{\delta}$

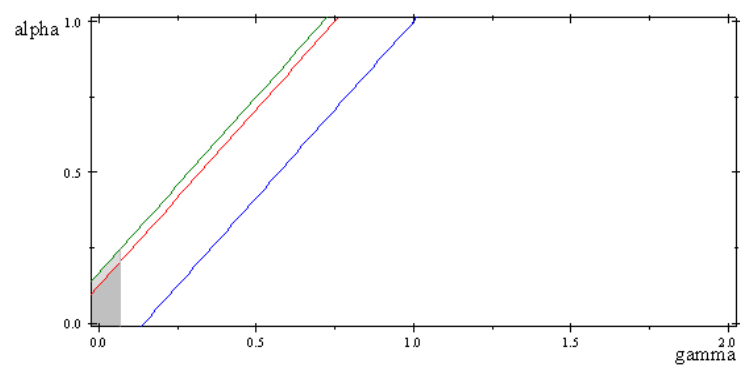

$\delta>6$ and $k \leq \frac{1}{\delta}$

$\frac{1}{k^{2}}\left(\int_{0}^{k} \int_{\frac{\delta}{\delta-1} \gamma+\frac{2-\delta k}{2(\delta-1)}}^{\frac{\delta}{\delta-1} \gamma+\frac{1}{\delta-1}} P D(\gamma, \alpha, \delta) d \alpha d \gamma+\int_{0}^{k} \int_{0}^{\frac{\delta}{\delta-1} \gamma+\frac{2-\delta k}{2(\delta-1)}} k d \alpha d \gamma\right)=\frac{k \delta+4}{4 \delta-4}$ 


\section{Case XV}

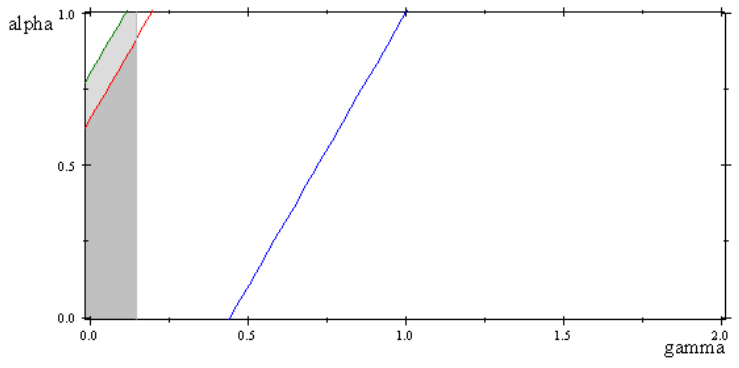

$2<\delta \leq \frac{5}{2}$ and $\frac{\delta-2}{\delta}<k \leq \frac{2 \delta-4}{\delta}$

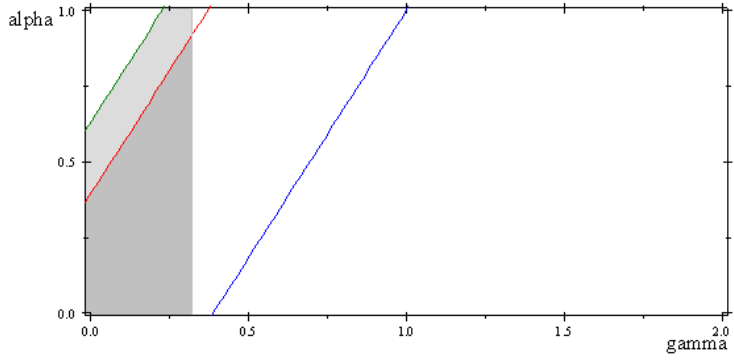

$\frac{5}{2}<\delta \leq 3$ and $\frac{\delta-2}{\delta}<k \leq \frac{1}{\delta}$

$$
\begin{aligned}
& \frac{1}{k^{2}}\left(\int_{0}^{\frac{\delta-2}{\delta}} \int_{\frac{\delta}{\delta-1} \gamma+\frac{2-\delta k}{2(\delta-1)}}^{\frac{\delta}{\delta-1} \gamma+\frac{1}{\delta-1}} P D(\gamma, \alpha, \delta) d \alpha d \gamma+\int_{\frac{\delta-2}{\delta}}^{k} \int_{\frac{\delta}{\delta-1} \gamma+\frac{2-\delta k}{2(\delta-1)}}^{1} P D(\gamma, \alpha, \delta) d \alpha d \gamma+\int_{0}^{k} \int_{0}^{\frac{\delta}{\delta-1} \gamma+\frac{2-\delta k}{2(\delta-1)}} k d \alpha d \gamma\right) \\
= & \frac{k^{3} \delta^{3}-12 k^{2} \delta^{3}+12 k^{2} \delta^{2}+12 k \delta^{3}-48 k \delta^{2}+48 k \delta-4 \delta^{3}+24 \delta^{2}-48 \delta+32}{12 k^{2} \delta^{2}(1-\delta)}
\end{aligned}
$$

\section{Case XVI}

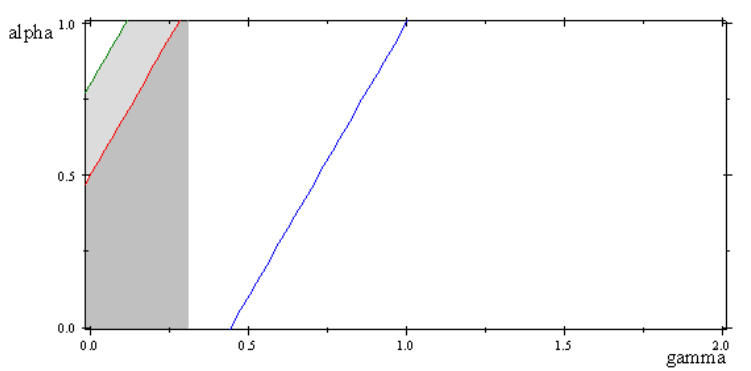

$$
2<\delta \leq \frac{5}{2} \text { and } \frac{2 \delta-4}{\delta}<k \leq \frac{1}{\delta}
$$

$$
\frac{1}{k^{2}} \int_{0}^{\frac{\delta-2}{\delta}} \int_{\frac{\delta}{\delta-1} \gamma+\frac{2-\delta k}{2(\delta-1)}}^{\frac{\delta}{\delta-1} \gamma+\frac{1}{\delta-1}} P D(\gamma, \alpha, \delta) d \alpha d \gamma+\frac{1}{k^{2}} \int_{\frac{\delta-2}{\delta}}^{\frac{2 \delta+k \delta-4}{2 \delta}} \int_{\frac{\delta}{\delta-1} \gamma+\frac{2-\delta k}{2(\delta-1)}}^{1} P D(\gamma, \alpha, \delta) d \alpha d \gamma+
$$

$+\frac{1}{k^{2}} \int_{0}^{\frac{2 \delta+k \delta-4}{2 \delta}} \int_{0}^{\frac{\delta}{\delta-1} \gamma+\frac{2-\delta k}{2(\delta-1)}} k d \alpha d \gamma+\frac{1}{k^{2}} \int_{\frac{2 \delta+k \delta-4}{2 \delta}}^{k} \int_{0}^{1} k d \alpha d \gamma=\frac{k^{2} \delta^{2}-18 k \delta^{2}+12 k \delta+12 \delta^{2}-48 \delta+48}{24 k \delta(1-\delta)}$

\section{Case XVII}

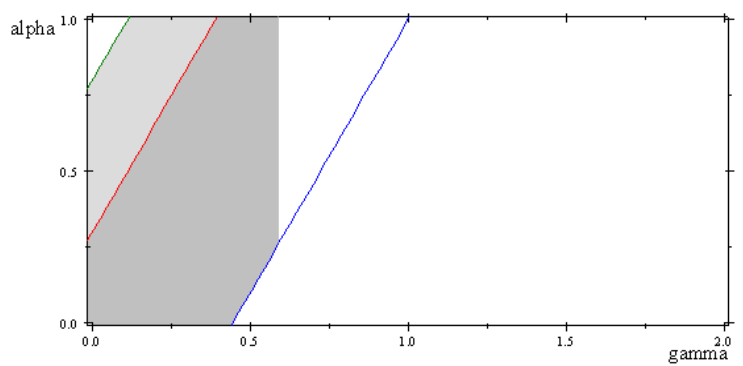

$2<\delta \leq \frac{5}{2}$ and $\frac{1}{\delta}<k \leq \frac{2}{\delta}$

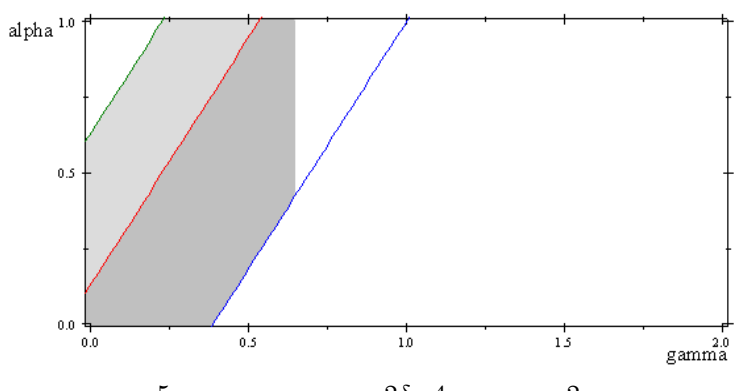

$\frac{5}{2}<\delta \leq 3$ and $\frac{2 \delta-4}{\delta}<k<\frac{2}{\delta}$ 


$$
\begin{aligned}
& \frac{1}{k^{2}} \int_{0}^{\frac{\delta-2}{\delta}} \int_{\frac{\delta}{\delta-1} \gamma+\frac{2-\delta k}{2(\delta-1)}}^{\frac{\delta}{\delta-1} \gamma+\frac{1}{\delta-1}} P D(\gamma, \alpha, \delta) d \alpha d \gamma+\frac{1}{k^{2}} \int_{\frac{\delta-2}{\delta}}^{\frac{2 \delta+k \delta-4}{2 \delta}} \int_{\frac{\delta}{\delta-1} \gamma+\frac{2-\delta k}{2(\delta-1)}}^{1} P D(\gamma, \alpha, \delta) d \alpha d \gamma+ \\
+ & \frac{1}{k^{2}} \int_{0}^{\frac{2 \delta \delta+k \delta-4}{2 \delta}} \int_{0}^{\frac{\delta}{\delta-1} \gamma+\frac{2-\delta k}{2(\delta-1)}} k d \alpha d \gamma+\frac{1}{k^{2}} \int_{\frac{2 \delta+k \delta-4}{2 \delta}}^{\frac{1}{\delta}} \int_{0}^{1} k d \alpha d \gamma+\int_{\frac{1}{\delta}}^{k} \int_{\frac{\delta}{\delta-1} \gamma-\frac{1}{\delta-1}}^{1} k d \alpha d \gamma= \\
= & \frac{13 k^{2} \delta^{2}-18 k \delta^{2}-12 k \delta+12 \delta^{2}-48 \delta+60}{24 k \delta(1-\delta)}
\end{aligned}
$$

\section{Case XVIII}
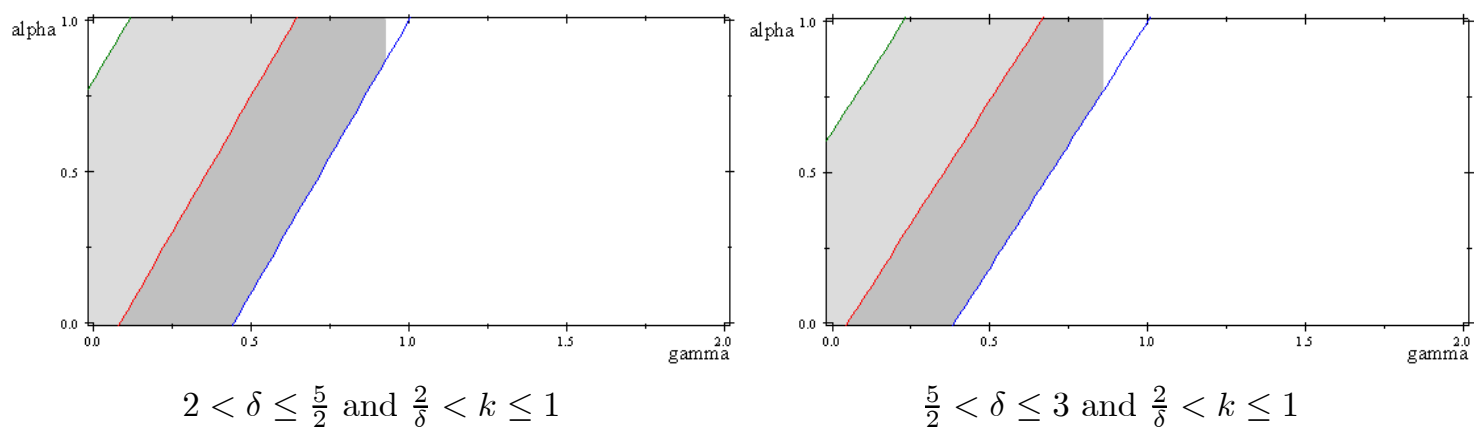

$2<\delta \leq \frac{5}{2}$ and $\frac{2}{\delta}<k \leq 1$

$\frac{5}{2}<\delta \leq 3$ and $\frac{2}{\delta}<k \leq 1$

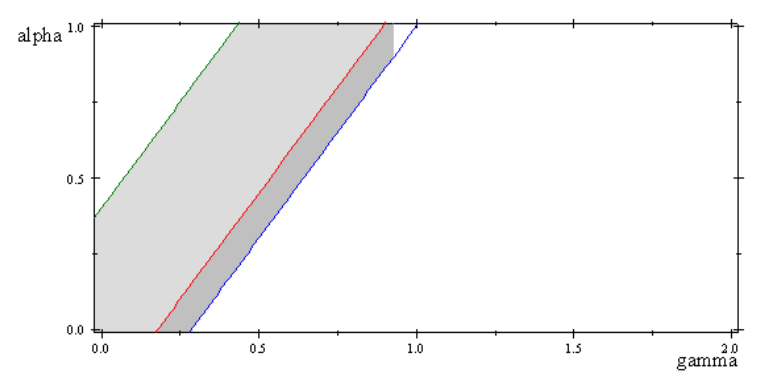

$$
3<\delta \leq 4 \text { and } \frac{2 \delta-4}{\delta}<k<1
$$

$$
\begin{aligned}
& \frac{1}{k^{2}} \int_{0}^{\frac{1}{\delta-1}} \int_{0}^{\frac{-2 \alpha+k \delta+2 \alpha \delta-2}{2 \delta}} P D(\gamma, \alpha, \delta) d \gamma d \alpha+\frac{1}{k^{2}} \int_{\frac{1}{\delta-1}}^{1} \int_{\frac{-1-\alpha+\alpha \delta}{\delta}}^{\frac{-2 \alpha+k \delta+2 \alpha \delta-2}{2 \delta}} P D(\gamma, \alpha, \delta) d \gamma d \alpha+ \\
+ & \frac{1}{k^{2}} \int_{0}^{\frac{\delta k-1}{\delta-1}} \int_{\frac{-2 \alpha+k \delta+2 \alpha \delta-2}{2 \delta}}^{\frac{1-\alpha+\alpha \delta}{\delta}} k d \gamma d \alpha+\frac{1}{k^{2}} \int_{\frac{\delta k-1}{\delta-1}}^{1} \int_{\frac{-2 \alpha+k \delta+2 \alpha \delta-2}{2 \delta}}^{k} k d \gamma d \alpha= \\
= & \frac{6 k^{3} \delta^{3}-9 k^{2} \delta^{3}-3 k^{2} \delta^{2}+6 k \delta^{3}-24 k \delta^{2}+24 k \delta+4}{12 k^{2} \delta^{2}(1-\delta)}
\end{aligned}
$$




\section{Case XIX}

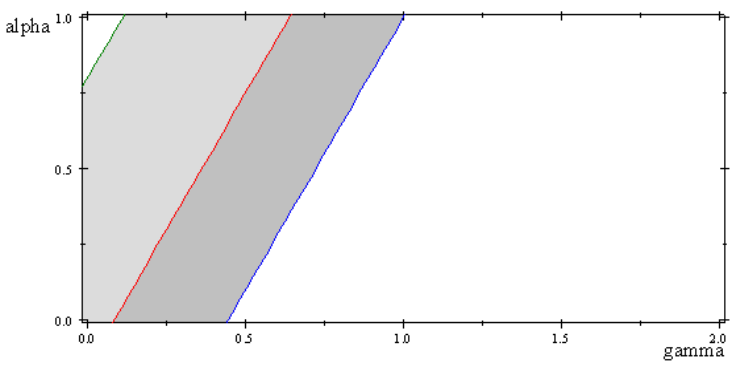

$2<\delta \leq \frac{5}{2}$ and $1<k \leq \frac{4}{\delta}$

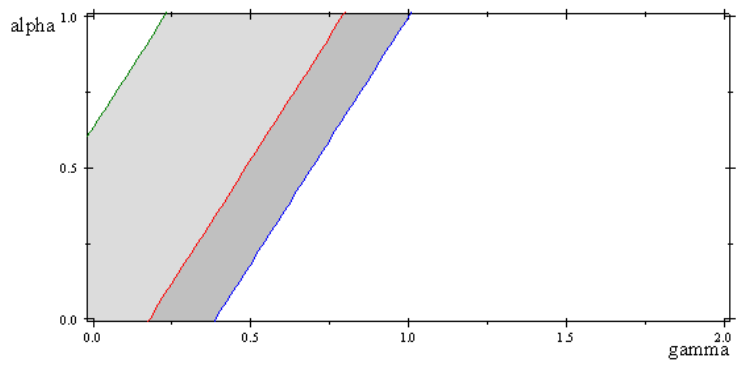

$\frac{5}{2}<\delta \leq 3$ and $1<k \leq \frac{4}{\delta}$

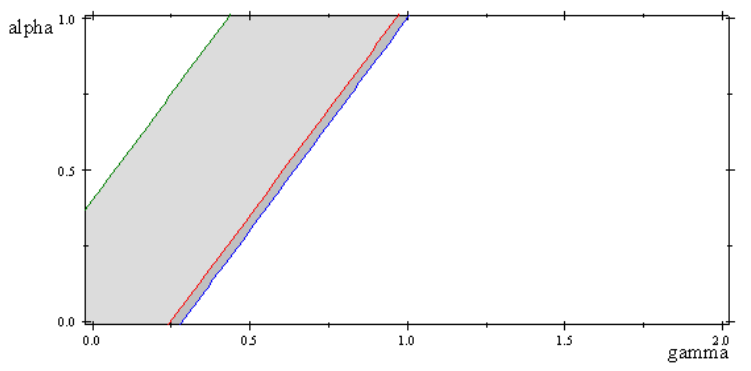

$3<\delta \leq 4$ and $1<k \leq \frac{4}{\delta}$

$\frac{1}{k^{2}} \int_{0}^{\frac{1}{\delta-1}} \int_{0}^{\frac{-2 \alpha+k \delta+2 \alpha \delta-2}{2 \delta}} P D(\gamma, \alpha, \delta) d \gamma d \alpha+\frac{1}{k^{2}} \int_{\frac{1}{\delta-1}}^{1} \int_{\frac{-1-\alpha+\alpha \delta}{\delta}}^{\frac{-2 \alpha+k \delta+2 \alpha \delta-2}{2 \delta}} P D(\gamma, \alpha, \delta) d \gamma d \alpha+$

$+\quad \frac{1}{k^{2}} \int_{0}^{1} \int_{\frac{-2 \alpha+k \delta+2 \alpha \delta-2}{2 \delta}}^{\frac{1-\alpha+\alpha \delta}{\delta}} k d \gamma d \alpha=\frac{3 k^{2} \delta^{3}-3 k^{2} \delta^{2}-24 k \delta^{2}+24 k \delta+4}{12 k^{2} \delta^{2}(1-\delta)}$

\section{Case XX}
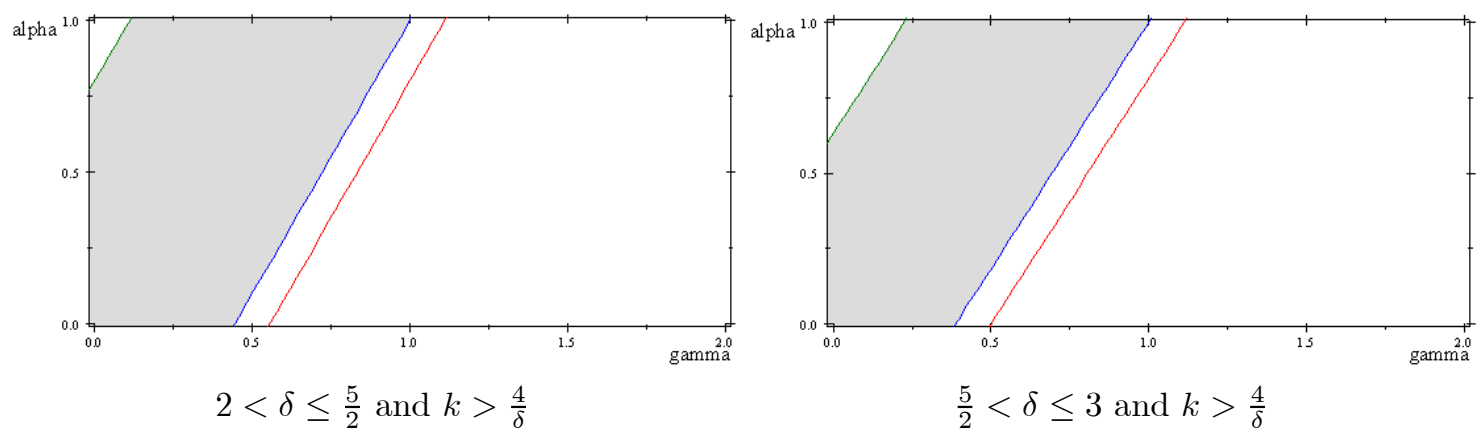


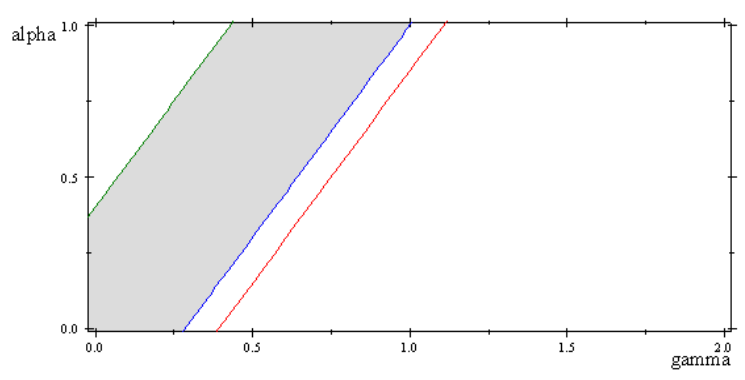

$3<\delta \leq 4$ and $k>\frac{4}{\delta}$

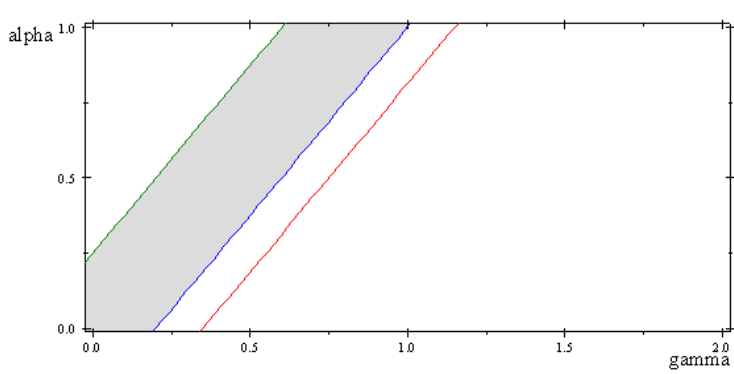

$4<\delta \leq 6$ and $k>1$

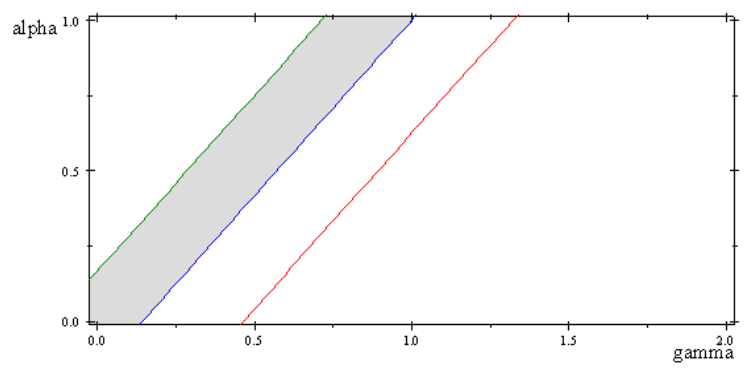

$$
\delta>6 \text { and } k>1
$$

$\frac{1}{k^{2}}\left(\int_{0}^{\frac{1}{\delta-1}} \int_{0}^{\frac{1-\alpha+\alpha \delta}{\delta}} P D(\gamma, \alpha, \delta) d \gamma d \alpha+\int_{\frac{1}{\delta-1}}^{1} \int_{\frac{-1-\alpha+\alpha \delta}{\delta}}^{\frac{1-\alpha+\alpha \delta}{\delta}} P D(\gamma, \alpha, \delta) d \gamma d \alpha\right)=\frac{-12 \delta+13}{3 k^{2} \delta^{2}-3 k^{2} \delta^{3}}$

\section{Case XXI}

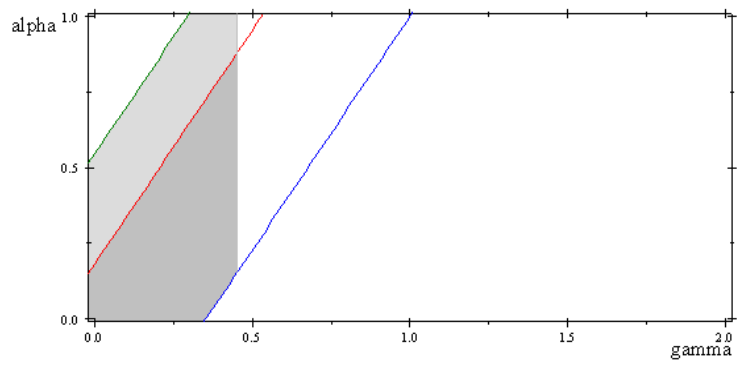

$\frac{5}{2}<\delta \leq 3$ and $\frac{1}{\delta}<k \leq \frac{2 \delta-4}{\delta}$

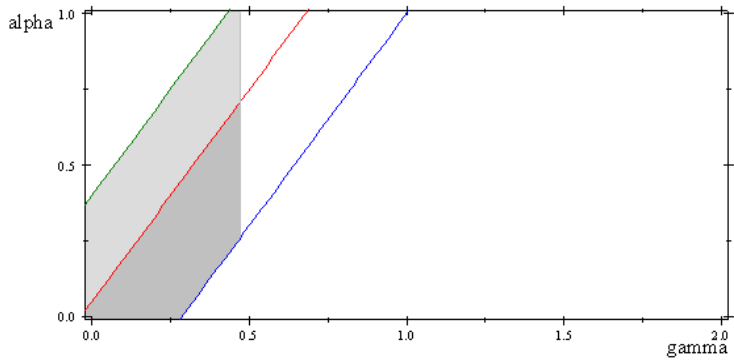

$3<\delta \leq 4$ and $\frac{\delta-2}{\delta}<k \leq \frac{2}{\delta}$

$$
\begin{aligned}
& \frac{1}{k^{2}} \int_{0}^{\frac{\delta-2}{\delta}} \int_{\frac{\delta}{\delta-1} \gamma+\frac{2-\delta k}{2(\delta-1)}}^{\frac{\delta}{\delta-1} \gamma+\frac{1}{\delta-1}} P D(\gamma, \alpha, \delta) d \alpha d \gamma+\frac{1}{k^{2}} \int_{\frac{\delta-2}{\delta}}^{k} \int_{\frac{\delta}{\delta-1} \gamma+\frac{2-\delta k}{2(\delta-1)}}^{1} P D(\gamma, \alpha, \delta) d \alpha d \gamma+ \\
+\quad & \frac{1}{k^{2}} \int_{0}^{\frac{1}{\delta}} \int_{0}^{\frac{\delta}{\delta-1} \gamma+\frac{2-\delta k}{2(\delta-1)}} k d \alpha d \gamma+\frac{1}{k^{2}} \int_{\frac{1}{\delta}}^{k} \int_{\frac{\delta}{\delta-1} \gamma-\frac{1}{\delta-1}}^{\frac{\delta}{\delta-1} \gamma+\frac{2-\delta k}{2(\delta-1)}} k d \alpha d \gamma= \\
= & \frac{7 k^{3} \delta^{3}-12 k^{2} \delta^{3}+12 k \delta^{3}-48 k \delta^{2}+54 k \delta-4 \delta^{3}+24 \delta^{2}-48 \delta+32}{12 k^{2} \delta^{2}(1-\delta)}
\end{aligned}
$$




\section{Case XXII}

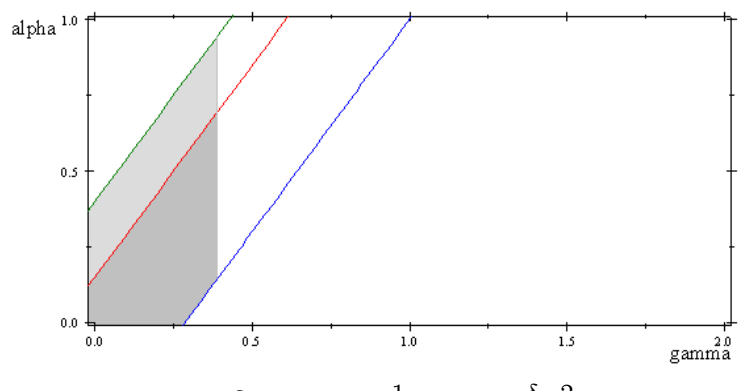

$3<\delta \leq 4$ and $\frac{1}{\delta}<k \leq \frac{\delta-2}{\delta}$

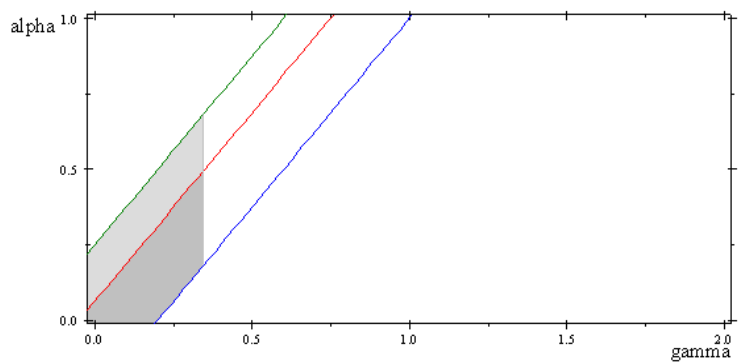

$4<\delta \leq 6$ and $\frac{1}{\delta}<k \leq \frac{2}{\delta}$

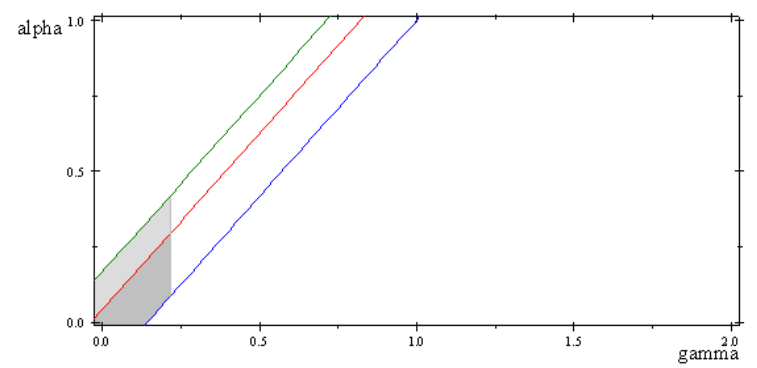

$\delta>6$ and $\frac{1}{\delta}<k \leq \frac{2}{\delta}$

$$
\begin{aligned}
& \frac{1}{k^{2}}\left(\int_{0}^{k} \int_{\frac{\delta}{\delta-1} \gamma+\frac{2-\delta k}{2(\delta-1)}}^{\frac{\delta}{\delta-1} \gamma+\frac{1}{\delta-1}} P D(\gamma, \alpha, \delta) d \alpha d \gamma+\int_{0}^{\frac{1}{\delta}} \int_{0}^{\frac{\delta}{\delta-1} \gamma+\frac{2-\delta k}{2(\delta-1)}} k d \alpha d \gamma+\int_{\frac{1}{\delta}}^{k} \int_{\frac{\delta}{\delta-1} \gamma-\frac{1}{\delta-1}}^{\frac{\delta}{\delta-1} \gamma+\frac{2-\delta k}{2(\delta-1)}} k d \alpha d \gamma\right)= \\
= & \frac{k^{2} \delta^{2}-8 k \delta+2}{4 k \delta(1-\delta)}
\end{aligned}
$$

\section{Case XXIII}

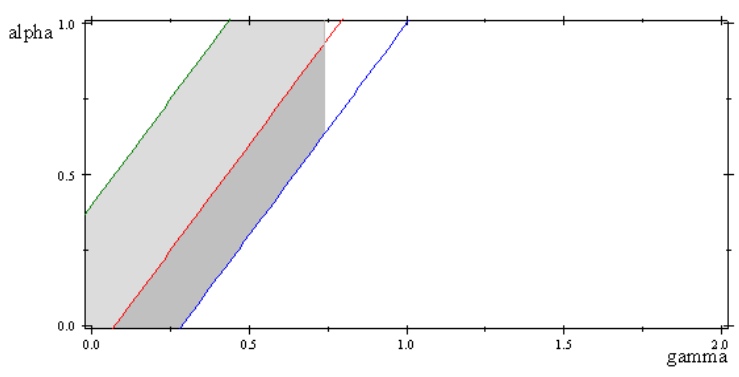

$3<\delta \leq 4$ and $\frac{2}{\delta}<k \leq \frac{2 \delta-4}{\delta}$

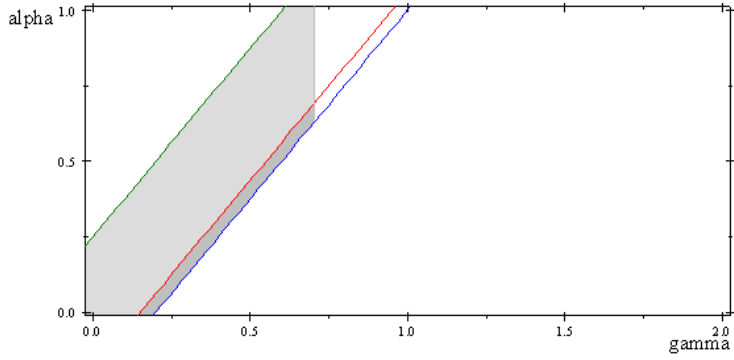

$4<\delta \leq 6$ and $\frac{\delta-2}{\delta}<k \leq \frac{4}{\delta}$

$$
\begin{aligned}
& \frac{1}{k^{2}} \int_{0}^{\frac{\delta k-2}{2 \delta}} \int_{0}^{\frac{\delta}{\delta-1} \gamma+\frac{1}{\delta-1}} P D(\gamma, \alpha, \delta) d \alpha d \gamma+\frac{1}{k^{2}} \int_{\frac{\delta k-2}{2 \delta}}^{\frac{\delta-2}{\delta}} \int_{\frac{\delta}{\delta-1} \gamma+\frac{2-\delta k}{2(\delta-1)}}^{\frac{\delta}{\delta-1} \gamma+\frac{1}{\delta-1}} P D(\gamma, \alpha, \delta) d \alpha d \gamma+ \\
+ & \frac{1}{k^{2}} \int_{\frac{\delta-2}{\delta}}^{k} \int_{\frac{\delta}{\delta-1} \gamma+\frac{2-\delta k}{2(\delta-1)}}^{1} P D(\gamma, \alpha, \delta) d \alpha d \gamma+\frac{1}{k^{2}} \int_{\frac{\delta k-2}{2 \delta}}^{\frac{1}{\delta}} \int_{0}^{\frac{\delta}{\delta-1} \gamma+\frac{2-\delta k}{2(\delta-1)}} k d \alpha d \gamma+\frac{1}{k^{2}} \int_{\frac{1}{\delta}}^{k} \int_{\frac{\delta}{\delta-1} \gamma-\frac{1}{\delta-1}}^{\frac{\delta}{\delta-1} \gamma+\frac{2-\delta k}{2(\delta-1)}} k d \alpha d \gamma= \\
= & \frac{13 k^{3} \delta^{3}-24 k^{2} \delta^{3}+6 k^{2} \delta^{2}+24 k \delta^{3}-96 k \delta^{2}+96 k \delta-8 \delta^{3}+48 \delta^{2}-96 \delta+72}{24 k^{2} \delta^{2}(1-\delta)}
\end{aligned}
$$




\section{Case XXIV}

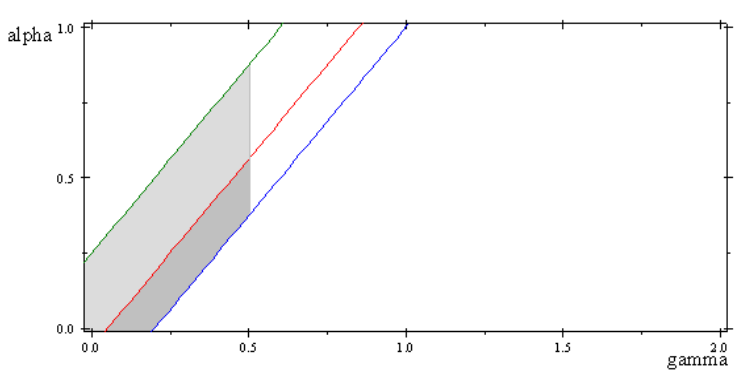

$4<\delta \leq 6$ and $\frac{2}{\delta}<k \leq \frac{\delta-2}{\delta}$

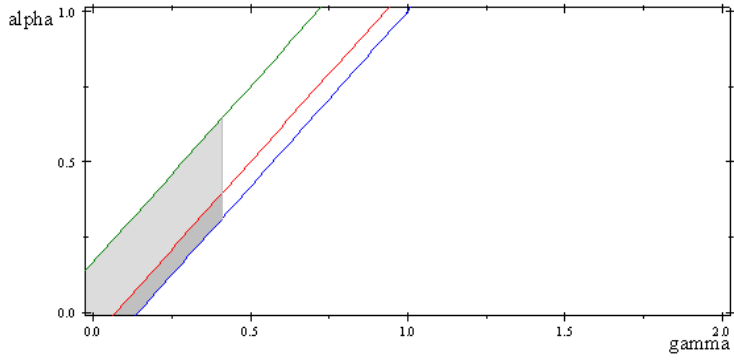

$\delta>6$ and $\frac{2}{\delta}<k \leq \frac{4}{\delta}$

$$
\begin{array}{r}
\frac{1}{k^{2}} \int_{0}^{\frac{\delta k-2}{2 \delta}} \int_{0}^{\frac{\delta}{\delta-1} \gamma+\frac{1}{\delta-1}} P D(\gamma, \alpha, \delta) d \alpha d \gamma+\frac{1}{k^{2}} \int_{\frac{\delta k-2}{2 \delta}}^{k} \int_{\frac{\delta}{\delta-1} \gamma+\frac{2-\delta k}{2(\delta-1)}}^{\frac{\delta}{\delta-1} \gamma+\frac{1}{\delta-1}} P D(\gamma, \alpha, \delta) d \alpha d \gamma+ \\
+\quad \frac{1}{k^{2}} \int_{\frac{\delta k-2}{2 \delta}}^{\frac{1}{\delta}} \int_{0}^{\frac{\delta}{\delta-1} \gamma+\frac{2-\delta k}{2(\delta-1)}} k d \alpha d \gamma+\frac{1}{k^{2}} \int_{\frac{1}{\delta}}^{k} \int_{\frac{\delta}{\delta-1} \gamma-\frac{1}{\delta-1}}^{\frac{\delta}{\delta-1} \gamma+\frac{2-\delta k}{2(\delta-1)}} k d \alpha d \gamma=\frac{5 k^{3} \delta^{3}-42 k^{2} \delta^{2}+8}{24 k^{2} \delta^{2}(1-\delta)}
\end{array}
$$

\section{Case XXV}

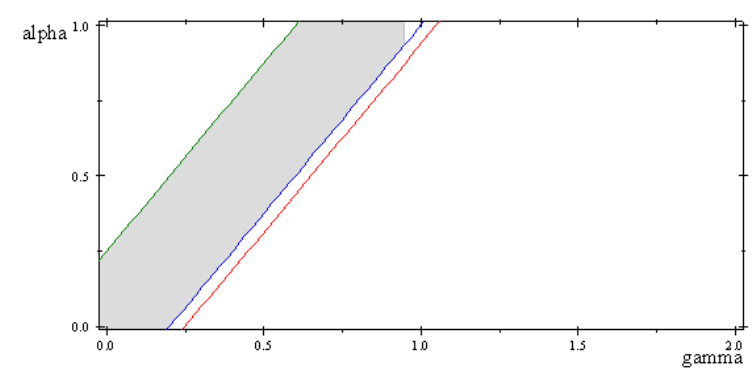

$4<\delta \leq 6$ and $\frac{4}{\delta}<k<1$

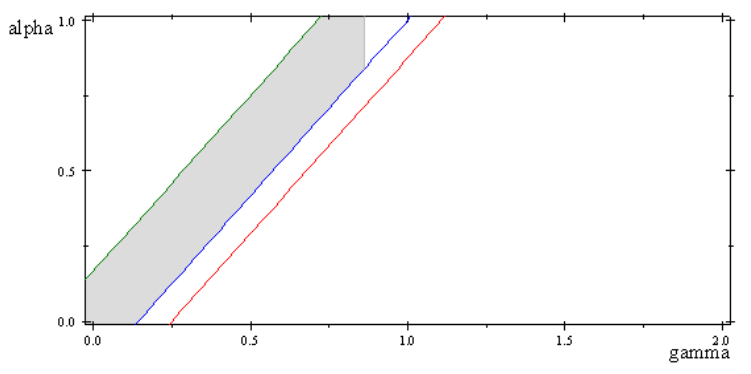

$\delta>6$ and $\frac{4}{\delta}<k \leq 1$

$$
\begin{aligned}
& \frac{1}{k^{2}} \int_{0}^{\frac{1}{\delta}} \int_{0}^{\frac{\delta}{\delta-1} \gamma+\frac{1}{\delta-1}} P D(\gamma, \alpha, \delta) d \alpha d \gamma+\frac{1}{k^{2}} \int_{\frac{1}{\delta}}^{\frac{\delta-2}{\delta}} \int_{\frac{\delta}{\delta-1} \gamma-\frac{1}{\delta-1}}^{\frac{\delta}{\delta-1} \gamma+\frac{1}{\delta-1}} P D(\gamma, \alpha, \delta) d \alpha d \gamma+ \\
+ & \frac{1}{k^{2}} \int_{\frac{\delta-2}{\delta}}^{k} \int_{\frac{\delta}{\delta-1} \gamma-\frac{1}{\delta-1}}^{1} P D(\gamma, \alpha, \delta) d \alpha d \gamma=\frac{k^{3} \delta^{3}-3 k^{2} \delta^{3}+6 k^{2} \delta^{2}+3 k \delta^{3}-12 k \delta^{2}-\delta^{3}+6 \delta^{2}-12 \delta+13}{3 k^{2} \delta^{2}-3 k^{2} \delta^{3}}
\end{aligned}
$$

\section{Qualitative analysis}

The probability function, $P(\delta, k)$, is now explicitly written. The following properties can be easily verified:

1. The function $P(\delta, k)$ is continuous.

2. The function $P(\delta, k)$ is decreasing with respect to $\delta$.

3. For each $\delta$ there is $k_{\delta}$ (with $k_{\delta}<1$ ) such that, for $k>k_{\delta}$, the function $P(\delta, k)$ is decreasing with respect to $k$. 
Figures 3 and 4 show a set of curves depicting the profiles of the function $P(\delta, k)$ (darkness decreases when increasing $k$, in fig. 3 , and $\delta$, in fig. 4) which illustrate the previous statements. The monotonicity with respect to $\delta$ can be explained by observing that such parameter measures, in some sense, the reactivity of the system. The higher the reactivity the lower the stability of the steady state. The parameter $k$ has, in a probabilistic sense, a similar effect. Indeed, increasing the value of $k$, the probability of obtaining - by a random draw from our uniform distribution - high values of $\beta$ and $\gamma$ also increases. In such a case the reactivity of price forecasts to small changes in the observed price may be high, reducing the stability of the system. On the contrary, the reason for the inversion in the monotonicity with respect to $k$ is not clear. One possible explanation is that certain amount of fundamentalist or trend chasing behavior is necessary to counter the inertia which, otherwise, dominates the dynamics and can lead to instability. This may happen when $\delta$ is big enough as suggested by the bifurcation diagrams in figures 1 and 2 and confirmed by the curves in figure 4 .

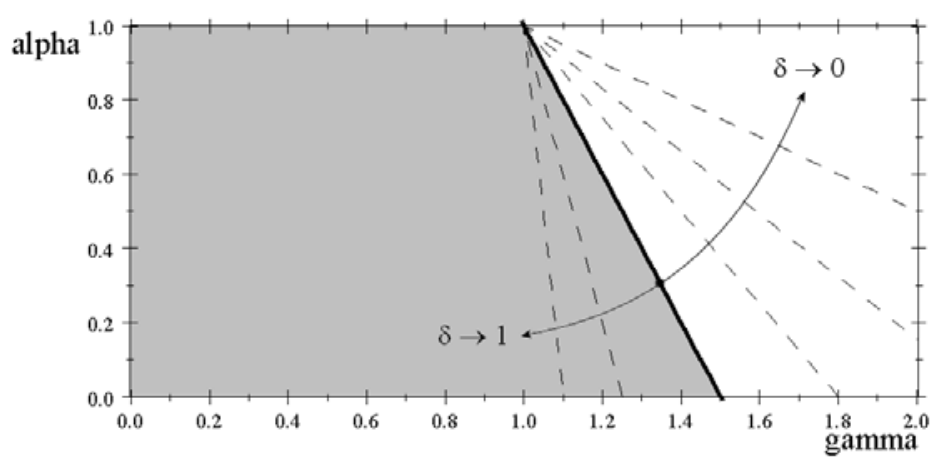

Figure 1: Bifurcation diagram as $\delta$ varies in $(0,1), \beta=\frac{5}{4}$

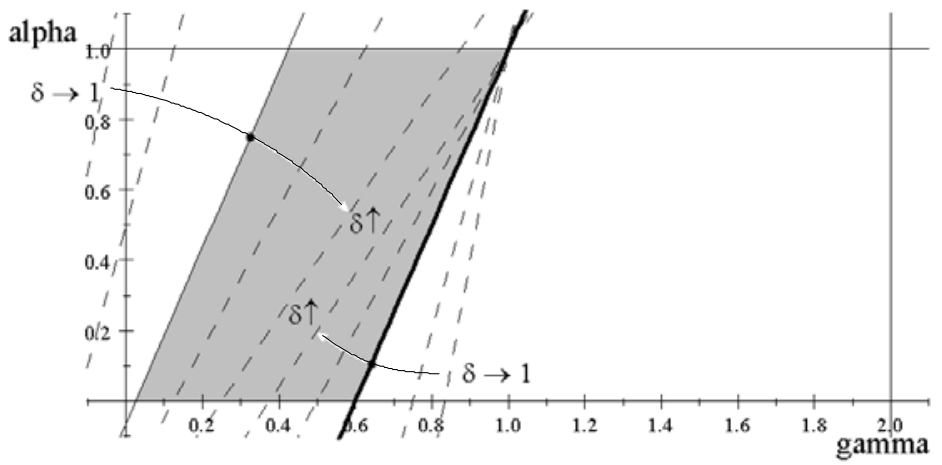

Figure 2: Bifurcation diagram for $\delta>1, \beta=\frac{5}{4}$ 


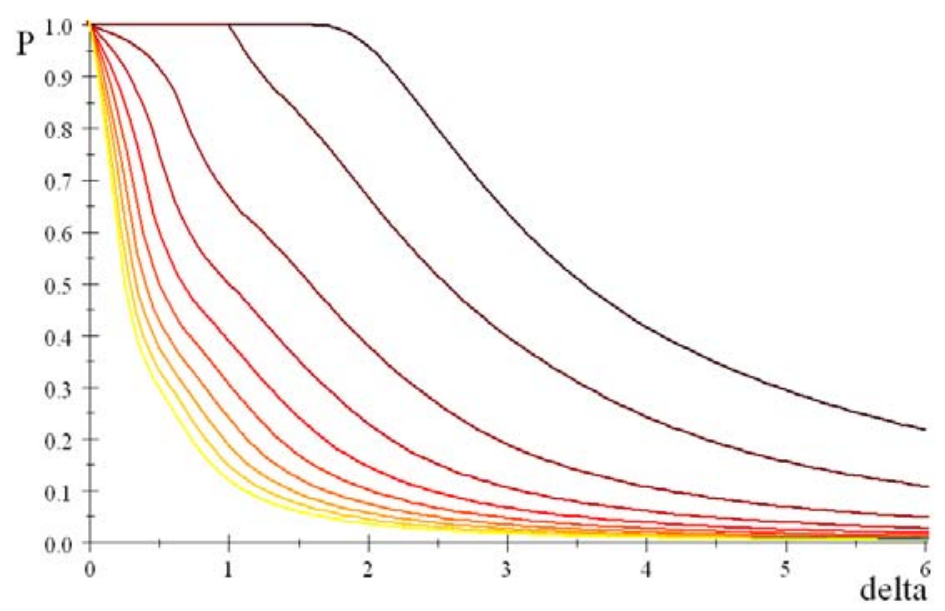

Figure 3: Profile of the function $P(\delta, k)$ for various $k$ (higher values for lighter colours)

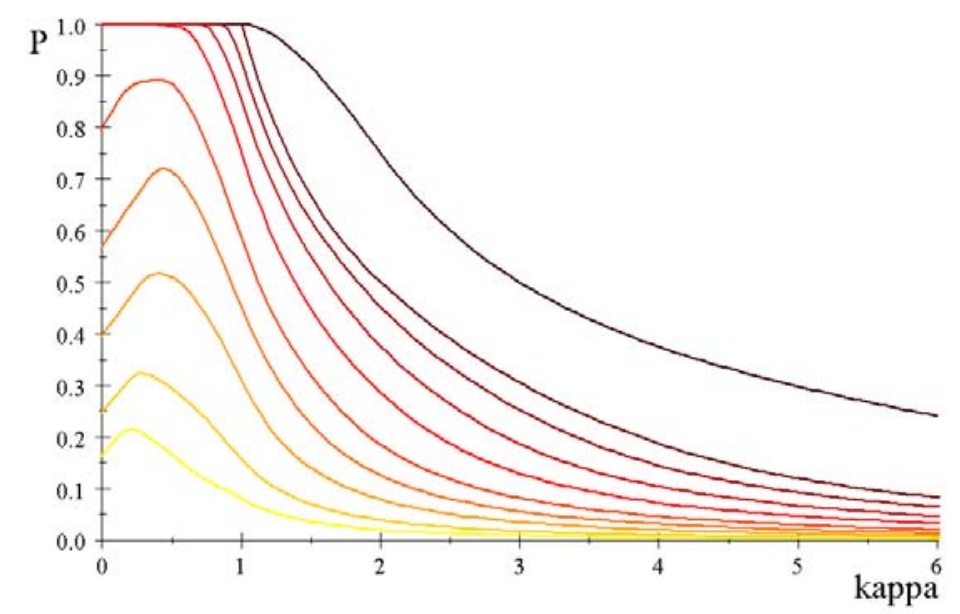

Figure 4: Profile of the function $P(\delta, k)$ for various $\delta$ (higher values for lighter colours) 